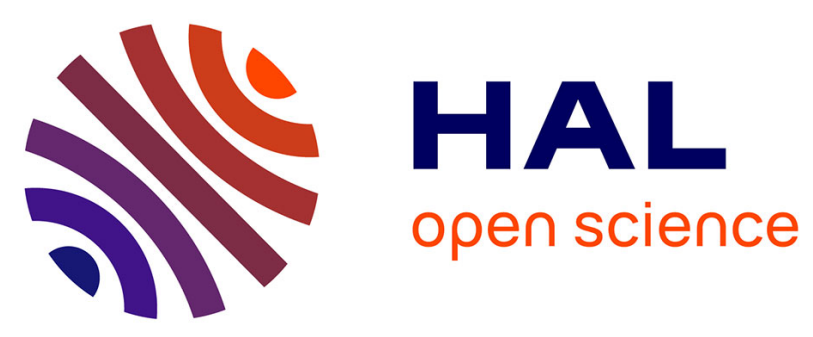

\title{
Adaptive inexact iterative algorithms based on polynomial-degree-robust a posteriori estimates for the Stokes problem
}

\author{
Martin Čermák, Frédéric Hecht, Zuqi Tang, Martin Vohralík
}

\section{- To cite this version:}

Martin Čermák, Frédéric Hecht, Zuqi Tang, Martin Vohralík. Adaptive inexact iterative algorithms based on polynomial-degree-robust a posteriori estimates for the Stokes problem. Numerische Mathematik, 2018, 138 (4), pp.1027-1065. 10.1007/s00211-017-0925-3 . hal-01097662v4

\section{HAL Id: hal-01097662 \\ https://inria.hal.science/hal-01097662v4}

Submitted on 20 Apr 2018

HAL is a multi-disciplinary open access archive for the deposit and dissemination of scientific research documents, whether they are published or not. The documents may come from teaching and research institutions in France or abroad, or from public or private research centers.
L'archive ouverte pluridisciplinaire HAL, est destinée au dépôt et à la diffusion de documents scientifiques de niveau recherche, publiés ou non, émanant des établissements d'enseignement et de recherche français ou étrangers, des laboratoires publics ou privés. 


\title{
Adaptive inexact iterative algorithms based on polynomial-degree-robust a posteriori estimates for the Stokes problem*
}

\author{
Martin Čermák $\quad$ Frédéric Hecht ${ }^{\S}$ Zuqi Tang* Martin Vohralík
}

\begin{abstract}
In this paper, we develop adaptive inexact versions of iterative algorithms applied to finite element discretizations of the linear Stokes problem. We base our developments on an equilibrated stress a posteriori error estimate distinguishing the different error components, namely the discretization error component, the (inner) algebraic solver error component, and possibly the outer algebraic solver error component for algorithms of the Uzawa type. We prove that our estimate gives a guaranteed upper bound on the total error, as well as a polynomial-degree-robust local efficiency, and this on each step of the employed iterative algorithm. Our adaptive algorithms stop the iterations when the corresponding error components do not have a significant influence on the total error. The developed framework covers all standard conforming and conforming stabilized finite element methods on simplicial and rectangular parallelepipeds meshes in two or three space dimensions and an arbitrary algebraic solver. Implementation into the FreeFem++ programming language is invoked and numerical examples showcase the performance of our a posteriori estimates and of the proposed adaptive strategies. As example, we choose here the unpreconditioned and preconditioned Uzawa algorithm and the preconditioned minimum residual (MinRes) algorithm, in combination with the Taylor-Hood discretization.
\end{abstract}

Key words: Stokes problem, conforming finite element method, adaptive inexact iterative algorithm, outer-inner iteration, Uzawa method, MinRes, a posteriori error estimate, guaranteed bound, efficiency, polynomial-degree-robustness, interplay between error components, adaptive stopping criterion

\section{Introduction}

Let $\Omega \subset \mathbb{R}^{d}, 2 \leq d \leq 3$, be an open bounded polygonal domain with Lipschitz boundary denoted by $\partial \Omega$. We consider in this paper the steady linear Stokes model problem: find a velocity $\mathbf{u}: \Omega \rightarrow \mathbb{R}^{d}$ and a pressure $p: \Omega \rightarrow \mathbb{R}$ satisfying:

$$
\begin{aligned}
-\Delta \mathbf{u}+\nabla p=\mathbf{f} & & \text { in } \Omega, \\
\nabla \cdot \mathbf{u}=0 & & \text { in } \Omega, \\
\mathbf{u}=\mathbf{0} & & \text { on } \partial \Omega,
\end{aligned}
$$

where $\mathbf{f}: \Omega \rightarrow \mathbb{R}^{d}$ represents a given volumetric force. The notations $\Delta, \nabla$, and $\nabla$. represent respectively the Laplacian, gradient, and divergence operators. Let

$$
\begin{aligned}
\mathbf{V} & :=\left[H_{0}^{1}(\Omega)\right]^{d}, \\
Q & :=L_{0}^{2}(\Omega):=\left\{q \in L^{2}(\Omega) ;(q, 1)=0\right\} .
\end{aligned}
$$

\footnotetext{
*This project has received funding from the European Research Council (ERC) under the European Unions Horizon 2020 research and innovation program (grant agreement No 647134 GATIPOR).

『 IT4Innovations national supercomputing center (IT4I), VSB-Technical University of Ostrava, 17. listopadu 15/2172, 70833 Ostrava - Poruba, Czech Republic (martin.cermak@vsb.cz).

§UPMC Univ Paris 06, UMR 7598, Laboratoire Jacques-Louis Lions, 75005, Paris, France \& CNRS, UMR 7598, Laboratoire Jacques-Louis Lions, 75005, Paris, France \& INRIA Paris, 2 rue Simone Iff, 75589 Paris 12, France (frederic.hecht@upmc.fr).

* GeePs (Group of electrical engineering-Paris), UMR CNRS 8507, CentraleSuplec, Univ. Paris-Sud, Université ParisSaclay, Sorbonne Universités, UPMC Univ Paris 06, 11 rue Joliot-Curie, Plateau de Moulon, 91192 Gif-sur-Yvette, France (zuqi.tang@geeps.centralesupelec.fr).

+INRIA Paris, 2 rue Simone Iff, 75589 Paris 12, France (martin.vohralik@inria.fr).
} 
The weak formulation of problem (1.1) reads (cf. Section 2 for the details on the notation):

Definition 1.1 (Weak solution). Find $(\mathbf{u}, p) \in \mathbf{V} \times Q$ such that

$$
\begin{aligned}
(\nabla \mathbf{u}, \nabla \mathbf{v})-(\nabla \cdot \mathbf{v}, p) & =(\mathbf{f}, \mathbf{v}) & & \forall \mathbf{v} \in \mathbf{V}, \\
-(\nabla \cdot \mathbf{u}, q) & =0 & & \forall q \in Q .
\end{aligned}
$$

Problem (1.3) is well-posed due to the inf-sup condition (we systematically assume the arguments nonzero)

$$
\inf _{q \in Q} \sup _{\mathbf{v} \in \mathbf{V}} \frac{(q, \nabla \cdot \mathbf{v})}{\|\nabla \mathbf{v}\|\|q\|}=\beta,
$$

where $\beta$ is a positive constant, only depending on the domain $\Omega$, cf. 33. In the case when the domain is simple, analytical results for the value of the constant $\beta$ are available, see for example [13, 41, 53]. Otherwise a numerical approximation is necessary. This subject is discussed for example in 22]. Alternatively, when a robust (spectrally-equivalent) preconditioning strategy is adopted in the numerical resolution, an estimate (possibly not guaranteed but quite precise in practice) of the inf-sup constant can be computed on-the-fly, which is for instant a key ingredient in the EST_MINRES algorithm of [51].

Alternatively, cf. [48, the velocity $\mathbf{u}$ is the unique solution of the problem: find $\mathbf{u} \in \mathbf{V}$ with $\nabla \cdot \mathbf{u}=0$ such that

$$
(\nabla \mathbf{u}, \nabla \mathbf{v})=(\mathbf{f}, \mathbf{v}) \quad \forall \mathbf{v} \in \mathbf{V} \text { with } \nabla \cdot \mathbf{v}=0 .
$$

Let $\mathbf{V}_{h} \subset \mathbf{V}$ and $Q_{h} \subset Q$ be two approximation spaces of piecewise polynomials on a mesh $\mathcal{T}_{h}$ of the domain $\Omega$; examples are given in Section 3 . We consider the discretization of problem (1.3) by the conforming finite element method, possibly stabilized when $t_{h}(\cdot, \cdot ; \cdot)$ or $s_{h}(\cdot, \cdot ; \cdot)$ below are nonzero:

Definition 1.2 ((Stabilized) finite element method). Find $\left(\mathbf{u}_{h}, p_{h}\right) \in \mathbf{V}_{h} \times Q_{h}$ such that

$$
\begin{aligned}
\left(\nabla \mathbf{u}_{h}, \nabla \mathbf{v}_{h}\right)-\left(\nabla \cdot \mathbf{v}_{h}, p_{h}\right)+t_{h}\left(\mathbf{u}_{h}, p_{h} ; \mathbf{v}_{h}\right) & =\left(\mathbf{f}, \mathbf{v}_{h}\right) & & \forall \mathbf{v}_{h} \in \mathbf{V}_{h}, \\
-\left(\nabla \cdot \mathbf{u}_{h}, q_{h}\right)+s_{h}\left(\mathbf{u}_{h}, p_{h} ; q_{h}\right) & =0 & & \forall q_{h} \in Q_{h} .
\end{aligned}
$$

Let $\left\{\mathbf{v}_{j}\right\}_{1 \leq j \leq M}$ be a basis of the space $\mathbf{V}_{h}$ and let $\left\{q_{j}\right\}_{1 \leq j \leq N}$ be a basis of the space $Q_{h}$. Using these basis functions in respectively 1.6a - 1.6b), we obtain a system of linear algebraic equations: find vectors $\mathrm{U} \in \mathbb{R}^{M}$ and $\mathrm{P} \in \mathbb{R}^{N}$ such that

$$
\left(\begin{array}{ll}
A & \mathbb{B} \\
\mathbb{C} & \mathbb{D}
\end{array}\right)\left(\begin{array}{l}
\mathrm{U} \\
\mathrm{P}
\end{array}\right)=\left(\begin{array}{l}
\mathrm{F} \\
\mathrm{G}
\end{array}\right)
$$

In this paper, we are interested in designing adaptive versions of common iterative algebraic solvers for the (approximate) solution of (1.7). The particularity of our approach is that we rely on an a posteriori estimate that gives a guaranteed bound on the overall error and distinguishes the different error components on each iteration step. We consider two classes of algorithms.

Outer-inner iterative algorithms, like the classical Uzawa method, see [6], are often developed in particular for the unstabilized methods, where the system (1.7) is indefinite but symmetric. They can be seen as a direct application of the gradient method to the minimization problem of the dual functional of the Stokes problem, see [7] for a general discussion. Applied to the general form (1.6), they can be abstractly written as, see [27, 43]:

Algorithm 1.3 (Outer-inner (Uzawa) algorithm).

1. Chose an initial approximation $p_{h}^{0} \in Q_{h}$ of $p, \mathbf{u}_{h}^{0,0} \in \mathbf{V}_{h}$ of $\mathbf{u}$, and a real constant $\alpha \in(0,2)$. Set $k:=-1$.

2. Do

(a) Increase $k:=k+1$.

(b) If $k \geq 1$, update $p_{h}^{k}:=p_{h}^{k-1}+\alpha \delta_{h}^{k-1, i}$. 
(c) Approximate $\mathbf{u}_{h}^{k, \infty} \in \mathbf{V}_{h}$, the solution to

$$
\left(\nabla \mathbf{u}_{h}^{k, \infty}, \nabla \mathbf{v}_{h}\right)+t_{h}\left(\mathbf{u}_{h}^{k, \infty}, p_{h}^{k} ; \mathbf{v}_{h}\right)=\left(\nabla \cdot \mathbf{v}_{h}, p_{h}^{k}\right)+\left(\mathbf{f}, \mathbf{v}_{h}\right) \quad \forall \mathbf{v}_{h} \in \mathbf{V}_{h}
$$

by an iterative algorithm, typically starting from $\mathbf{u}_{h}^{k, 0}$ (given by $\mathbf{u}_{h}^{k-1, i}$ if $k \geq 1$ ): set $i:=0$ and Do

$i$. Increase $i:=i+1$.

ii. Obtain the approximation $\mathbf{u}_{h}^{k, i} \in \mathbf{V}_{h}$ to (1.8).

iii. Set up the residual equation, yielding $\mathrm{R}^{k, i} \in \mathbb{R}^{M}$ satisfying

$$
\begin{array}{r}
\left(\nabla \mathbf{u}_{h}^{k, i}, \nabla \mathbf{v}_{j}\right)+t_{h}\left(\mathbf{u}_{h}^{k, i}, p_{h}^{k} ; \mathbf{v}_{j}\right)=\left(\nabla \cdot \mathbf{v}_{j}, p_{h}^{k}\right)+ \\
\left(\mathbf{f}, \mathbf{v}_{j}\right)-\mathrm{R}_{j}^{k, i} \\
\forall 1 \leq j \leq M .
\end{array}
$$

while

$$
\mathrm{R}^{k, i} \text { is not sufficiently small. }
$$

(d) Compute $\delta_{h}^{k, i} \in Q_{h}$ via

$$
\left(\delta_{h}^{k, i}, q_{h}\right)=\left(\nabla \cdot \mathbf{u}_{h}^{k, i}, q_{h}\right)-s_{h}\left(\mathbf{u}_{h}^{k, i}, p_{h}^{k} ; q_{h}\right), \quad \forall q_{h} \in Q_{h} .
$$

while

$$
\delta_{h}^{k, i} \alpha \text { is not sufficiently small. }
$$

Various works are dedicated to the choice of the parameter $\alpha$, e.g., [27, 7] and the references therein. The most attractive feature of the Uzawa method is its simplicity of implementation and its minimal memory requirements, though its speed of convergence is typically slow.

The structure of the algebraic system (1.7) also allows for efficient use of single-loop algebraic solvers; in particular, the minimum residual method (MinRes) by Paige \& Saunders 45] is very popular when (1.7) is symmetric. A general single-loop iterative algorithm for problem (1.6) writes:

Algorithm 1.4 (Arbitrary iterative (single-loop) algorithm).

1. Chose algebraic solver starting approximations $p_{h}^{0} \in Q_{h}$ and $\mathbf{u}_{h}^{0} \in \mathbf{V}_{h}$. Set $i:=0$.

2. Do

(a) Increase $i:=i+1$.

(b) Compute $\left(\mathbf{u}_{h}^{i}, p_{h}^{i}\right) \in \mathbf{V}_{h} \times Q_{h}$.

(c) Set up the residual equations, yielding $\mathrm{R}_{\mathbf{u}}^{i} \in \mathbb{R}^{M}$ and $\mathrm{R}_{p}^{i} \in \mathbb{R}^{N}$ with

$$
\begin{array}{rr}
\left(\nabla \mathbf{u}_{h}^{i}, \nabla \mathbf{v}_{j}\right)+t_{h}\left(\mathbf{u}_{h}^{i}, p_{h}^{i} ; \mathbf{v}_{j}\right)=\left(\nabla \cdot \mathbf{v}_{j}, p_{h}^{i}\right)+\left(\mathbf{f}, \mathbf{v}_{j}\right)-\mathrm{R}_{\mathbf{u}, j}^{i} \\
\forall 1 \leq j \leq M, \\
-\left(\nabla \cdot \mathbf{u}_{h}^{i}, q_{j}\right)+s_{h}\left(\mathbf{u}_{h}^{i}, p_{h}^{i} ; q_{j}\right)=-\mathrm{R}_{p, j}^{i} & \\
\forall 1 \leq j \leq N .
\end{array}
$$

while

$\mathrm{R}_{\mathbf{u}}^{i}$ and $\mathrm{R}_{p}^{i}$ are not sufficiently small.

Algorithm 1.3 necessitates practical stopping criteria in both the inner and outer loops, and Algorithm 1.4 needs a stopping criterion in its single (outer) loop. The Uzawa inner loop stopping criterion is typically treated on the algebraic level only, i.e., the closeness of $\mathbf{u}_{h}^{k, i}$ to the solution $\mathbf{u}_{h}^{k, \infty}$ of equation (1.8) is studied without reference to the underlying continuous Stokes problem $\sqrt{1.3}$. This gives rise to the inexact Uzawa method, see 56, 27, 15, 20, 7, 43, 42 and the references therein. To stop the outer loops, though, a link to the discretization error of (1.6) with respect to 1.3 appears to be a central point. One of the first contributions balancing algebraic and discretization errors in the Stokes setting is the work of Becker [10. Later, Silvester and Simoncini [51] derived stopping criteria for the (preconditioned) MinRes algorithm and Anjam et al. 3] estimated the total error at each Uzawa step for the exact Uzawa algorithm. Here, one is typically lead to build on a posteriori error estimates obtained for the Stokes problem with an exact algebraic solver; some of the main references here are [55, 8, 2, 39, 19, 50, 57, 1, 52. Adaptive stopping 
criteria via a posteriori error estimates in the context of other model problems are treated in [11, 44, 49, 4], see also the references therein.

In Section 4 of this paper, we first design adaptive versions of the above algebraic algorithms. We propose in particular adaptive stopping criteria in 1.10 and $\sqrt{1.12}$ of Algorithm 1.3 , as well as in (1.14) of Algorithm 1.4. All our criteria are of the same nature, requesting a balance of the (inner and outer) algebraic error component(s) with the discretization error. We extend to the Stokes setting the approach of [38, 29] whose salient property is that estimates on all error components come with the same multiplicative constant equal to one, so that no artificial mutual numerical scaling is introduced. This is possible thanks to the concept of the equilibrated stress reconstruction that we detail in Section 5. We follow the spirit of the unified framework of [34, but extend to the Stokes setting the methodology of [21, 14, 29, 30, 31. Remarkably, we obtain in Theorem 6.1 and Corollary 6.2 of Section 6 a guaranteed upper bound on the

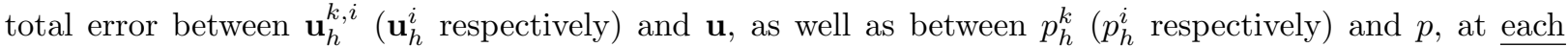
step of the algorithms. Under appropriate (local) stopping criteria, this crucially allows us to prove a polynomial-degree-robust (local) efficiency in Theorem 7.1 of Section 7 , and this in the presence of different error components.

Our results are presented in an abstract framework. Their application merely necessitates the verification of the sole Assumption 3.1 which is straightforward for any conforming or conforming stabilized finite element discretization (see the examples in Section 3), any iterative algebraic solver like those of $\overline{\text { Algo- }}$ rithms 1.3 and 1.4 and an arbitrary preconditioning. Section 8 presents several numerical examples, where, as representatives, we use Taylor-Hood unstabilized finite elements, the Uzawa and the MinRes algorithms, and no or incomplete Cholesky factorization preconditioners. We consider in our development homogeneous Dirichlet boundary conditions for simplicity only; inhomogeneous Dirichlet \& Neumann conditions can be taken into account as in [24, 31].

\section{Notation}

This section fixes the basic notation used throughout the paper.

Let $\mathbf{u}=\left[\mathbf{u}_{m}\right]_{1 \leq m \leq d}, \mathbf{v}=\left[\mathbf{v}_{m}\right]_{1 \leq m \leq d}$ denote vector functions and $\mathbb{u}=\left[\mathbf{u}_{m, n}\right]_{1 \leq m, n \leq d}, \mathbb{v}=\left[\mathbb{v}_{m, n}\right]_{1 \leq m, n \leq d}$ denote tensor functions. The respective scalar products are defined as $\mathbf{u} \cdot \mathbf{v}:=\sum_{m=1}^{d} \mathbf{u}_{m} \mathbf{v}_{m}$ and $\mathbb{u}: \mathbb{v}:=$ $\sum_{m=1}^{d} \sum_{n=1}^{d} \mathbb{u}_{m, n} \mathbb{v}_{m, n}$. We follow the convention that

$$
\nabla \mathbf{u}=\left(\begin{array}{ccc}
\frac{\partial \mathbf{u}_{1}}{\partial \mathbf{x}_{1}}, & \ldots, & \frac{\partial \mathbf{u}_{1}}{\partial \mathbf{x}_{d}} \\
\vdots & \ddots & \vdots \\
\frac{\partial \mathbf{u}_{d}}{\partial \mathbf{x}_{1}}, & \ldots, & \frac{\partial \mathbf{u}_{d}}{\partial \mathbf{x}_{d}}
\end{array}\right), \quad \nabla \cdot \mathbb{v}=\left(\begin{array}{ccc}
\frac{\partial \mathbb{v}_{1,1}}{\partial \mathbf{x}_{1}} & +\ldots & +\frac{\partial \mathbb{v}_{1, d}}{\partial \mathbf{x}_{d}} \\
\vdots & \ddots & \vdots \\
\frac{\partial \mathbb{v}_{d, 1}}{\partial \mathbf{x}_{1}} & +\ldots & +\frac{\partial \mathbb{v}_{d, d}}{\partial \mathbf{x}_{d}}
\end{array}\right)
$$

and

$$
\nabla \cdot \mathbf{u}=\sum_{m=1}^{d} \frac{\partial \mathbf{u}_{m}}{\partial \mathbf{x}_{m}} .
$$

Let $D \subset \mathbb{R}^{d}$; we denote by $(p, q)_{D}$ the integral $\int_{D} p q \mathrm{~d} \mathbf{x}$, where $p, q \in L^{2}(D)$. We use the same symbol $(\cdot, \cdot)_{D}$ also for the integrals of vector functions with values in $\mathbb{R}^{d}$ and of tensor functions with values in $\mathbb{R}^{d \times d}$, with the above scalar products. The Lebesgue norm on $L^{2}(D)$ is denoted by $\|\cdot\|_{D}$, respectively, and alike for vectors and tensors. When the domain $D$ coincides with $\Omega$, the subscript $\Omega$ is dropped. The same symbol $\|\cdot\|$ will also be used for the Euclidean norm of a vector $\mathrm{V} \in \mathbb{R}^{N}$,

$$
\|\mathrm{V}\|^{2}:=\sum_{m=1}^{N}\left(\mathrm{~V}_{m}\right)^{2}
$$

Moreover, we denote by $\|\cdot\|_{\infty}$ the infinity norm.

Let $\mathcal{T}_{h}$ be a mesh of $\Omega$, matching in the sense that for two distinct elements of $\mathcal{T}_{h}$, their intersection is either an empty set or their common vertex, edge, or face. A generic mesh element is either a $d$-dimensional 
simplex, or a $d$-dimensional rectangular parallelepiped. For $l \geq 0$, let $\mathbb{P}_{l}\left(\mathcal{T}_{h}\right)$ denote piecewise polynomials with respect to $\mathcal{T}_{h}$ of total degree at most $l$, and $\mathbb{Q}_{l}\left(\mathcal{T}_{h}\right)$ piecewise polynomials of degree at most $l$ in each variable. We let $\Pi_{l}$ be the $L^{2}(\Omega)$-orthogonal projection onto $\mathbb{P}_{l}\left(\mathcal{T}_{h}\right)$ or $\mathbb{Q}_{l}\left(\mathcal{T}_{h}\right) ; \Pi_{l}$ stands for this projector componentwise. We let $h_{K}$ denote the diameter of $K \in \mathcal{T}_{h}$ and use $h_{\Omega}$ for the diameter of the domain $\Omega$. Define the shape regularity parameter $\kappa_{\mathcal{T}_{h}}$ by

$$
\kappa_{\mathcal{T}_{h}}:=\max _{K \in \mathcal{T}_{h}} \frac{h_{K}}{\rho_{K}},
$$

where $\rho_{K}$ represents the diameter of the largest circle inscribed into the element $K$. We suppose henceforth that there exists a real number $\kappa_{\mathcal{T}}>0$ which bounds $\kappa_{\mathcal{T}_{h}}$ for any considered mesh $\mathcal{T}_{h}$.

We let $\mathcal{E}_{h}^{\text {int }}$ be the set of all interior faces denoted by $e ; \llbracket \cdot \rrbracket$ is then the jump operator across $e$. We will below also denote by $\mathcal{V}_{h}$ the set of vertices of $\mathcal{T}_{h}, \mathcal{V}_{h}^{\text {int }}$ for the set of interior vertices, and $\mathcal{V}_{h}^{\text {ext }}$ for the set of boundary vertices. For each $\mathbf{a} \in \mathcal{V}_{h}$, let $\omega_{\mathbf{a}}$ denote the patch of the vertex a, i.e., all the elements $K \in \mathcal{T}_{h}$ which share the vertex $\mathbf{a}$, and let $h_{\omega_{\mathbf{a}}}$ denote the diameter of the patch $\omega_{\mathbf{a}}$. Let $\psi_{\mathbf{a}} \in \mathbb{P}_{1}\left(\mathcal{T}_{h}\right) \cap C^{0}(\bar{\Omega})$ or $\psi_{\mathbf{a}} \in \mathbb{Q}_{1}\left(\mathcal{T}_{h}\right) \cap C^{0}(\bar{\Omega})$ stand for the hat basis function associated with the vertex $\mathbf{a} \in \mathcal{V}_{h}$, i.e., $\psi_{\mathbf{a}}$ is piecewise affine or piecewise $d$-affine with respect to the mesh $\mathcal{T}_{h}, \psi_{\mathbf{a}}(\mathbf{a})=1$ and $\psi_{\mathbf{a}}\left(\mathbf{a}^{\prime}\right)=0$ for $\mathbf{a}^{\prime} \in \mathcal{V}_{h}, \mathbf{a}^{\prime} \neq \mathbf{a}$. Let $\boldsymbol{\psi}_{\mathbf{a}, m}, 1 \leq m \leq d$ be its vector variants with $\left(\boldsymbol{\psi}_{\mathbf{a}, m}\right)_{n}=\psi_{\mathbf{a}} \delta_{m n}, 1 \leq n \leq d, \delta_{m n}=1$ if $m=n$ and 0 otherwise.

\section{Conforming finite element methods}

We shall derive our results in a quite general framework, taking into account all methods of the form of Definition 1.2 that satisfy the following assumption:

Assumption 3.1 (Admissible finite element discretization). The discretization stems from Definition 1.2 where:

1. $\mathbf{V}_{h}$ contains continuous piecewise affine or continuous piecewise d-affine functions and there exists an integer $l \geq 1$ such that $\mathbf{V}_{h}$ and $Q_{h}$ are respectively contained in discontinuous piecewise polynomials of maximal degree $l$ and $l-1$ :

$$
\begin{aligned}
& \mathbf{V} \cap\left[\mathbb{P}_{1}\left(\mathcal{T}_{h}\right)\right]^{d} \subset \mathbf{V}_{h} \subset\left[\mathbb{P}_{l}\left(\mathcal{T}_{h}\right)\right]^{d}, \quad Q_{h} \subset \mathbb{P}_{l-1}\left(\mathcal{T}_{h}\right) \quad \text { on simplices, } \\
& \mathbf{V} \cap\left[\mathbb{Q}_{1}\left(\mathcal{T}_{h}\right)\right]^{d} \subset \mathbf{V}_{h} \subset\left[\mathbb{Q}_{l}\left(\mathcal{T}_{h}\right)\right]^{d}, \quad Q_{h} \subset \mathbb{Q}_{l-1}\left(\mathcal{T}_{h}\right) \quad \text { on rect. par. }
\end{aligned}
$$

2. The stabilization term $t_{h}(\cdot, \cdot ; \cdot)$ vanishes over the hat test functions:

$$
t_{h}\left(\mathbf{v}_{h}, q_{h} ; \boldsymbol{\psi}_{\mathbf{a}, m}\right)=0 \quad \forall\left(\mathbf{v}_{h}, q_{h}\right) \in \mathbf{V}_{h} \times Q_{h}, \mathbf{a} \in \mathcal{V}_{h}^{\text {int }}, 1 \leq m \leq d .
$$

Note in particular that Assumption 3.1 implies from Definition 1.2 that

$$
\left(\nabla \mathbf{u}_{h}, \nabla \boldsymbol{\psi}_{\mathbf{a}, m}\right)-\left(\nabla \cdot \boldsymbol{\psi}_{\mathbf{a}, m}, p_{h}\right)=\left(\mathbf{f}, \boldsymbol{\psi}_{\mathbf{a}, m}\right) \quad \forall \mathbf{a} \in \mathcal{V}_{h}^{\text {int }}, 1 \leq m \leq d
$$

for an exact solve algebraic solve of the system (1.7). Indeed, this follows immediately by taking $\boldsymbol{\psi}_{\mathbf{a}, m}$ as the test functions in (1.6a). This hat function orthogonality is a crucial property for building the stress reconstruction in Definition 5.1 below and consequently for the guaranteed reliability of Theorem 6.1. The inclusion of the spaces $\mathbf{V}_{h}$ and $Q_{h}$ into piecewise polynomials in $3.1 \mathrm{a}$ is then central for the polynomialdegree-robust efficiency of Theorem 7.1 below.

Assumption 3.1 can be satisfied (choosing in particular sufficiently high polynomial degree $l$ for bubbleenriched methods) for all standard conforming finite element discretizations of the form of Definition 1.2 In order to fix ideas, we now give a (none-exhaustive) list of examples, cf. [48, 13, 28, for a general reference.

\subsection{Unstabilized schemes}

Definition 1.2 can in particular be considered with $s_{h}\left(\mathbf{u}_{h}, p_{h} ; q_{h}\right)$ and $t_{h}\left(\mathbf{u}_{h}, p_{h} ; q_{h}\right)$ equal to zero and the spaces $\mathbf{V}_{h} \times Q_{h}$ given by: 
- The Taylor-Hood family [54, 17, for $l \geq 2$,

$$
\begin{array}{lll}
\mathbf{V}_{h}:=\mathbf{V} \cap\left[\mathbb{P}_{l}\left(\mathcal{T}_{h}\right)\right]^{d}, & Q_{h}:=Q \cap\left[C^{0}(\bar{\Omega}) \cap \mathbb{P}_{l-1}\left(\mathcal{T}_{h}\right)\right] & \text { on simplices, } \\
\mathbf{V}_{h}:=\mathbf{V} \cap\left[\mathbb{Q}_{l}\left(\mathcal{T}_{h}\right)\right]^{d}, & Q_{h}:=Q \cap\left[C^{0}(\bar{\Omega}) \cap \mathbb{Q}_{l-1}\left(\mathcal{T}_{h}\right)\right] & \text { on rect. par. }
\end{array}
$$

- The mini element $[5$

$$
\mathbf{V}_{h}:=\mathbf{V} \cap\left[\mathbb{P}_{1}^{b}\left(\mathcal{T}_{h}\right)\right]^{d}, \quad Q_{h}:=Q \cap\left[C^{0}(\bar{\Omega}) \cap \mathbb{P}_{1}\left(\mathcal{T}_{h}\right)\right] \quad \text { on simplices },
$$

where $\mathbb{P}_{1}^{b}\left(\mathcal{T}_{h}\right)$ stands for piecewise affine functions from $\mathbb{P}_{1}\left(\mathcal{T}_{h}\right)$ enriched by the bubble functions vanishing on the boundary of each element. We need to set here the index $l:=d+1$.

- The cross-grid $\mathbb{P}_{1}-\mathbb{P}_{1}$ element [47, with $l=1$,

$$
\mathbf{V}_{h}:=\mathbf{V} \cap\left[\mathbb{P}_{1}\left(\mathcal{T}_{h}\right)\right]^{d}, \quad Q_{h}:=Q \cap\left[C^{0}(\bar{\Omega}) \cap \mathbb{P}_{1}\left(\mathcal{T}_{H}\right)\right] \quad \text { on simplices },
$$

where the simplicial mesh $\mathcal{T}_{h}$ was formed from the simplicial mesh $\mathcal{T}_{H}$ by adding the barycenter as a node and cutting each simplex into $(\mathrm{d}+1)$ subsimplices.

- The $\mathbb{P}_{1}$ iso $\mathbb{P}_{2}-\mathbb{P}_{1}$ or $\mathbb{Q}_{1}$ iso $\mathbb{Q}_{2}-\mathbb{Q}_{1}$ element [12], with $l=1$,

$$
\begin{array}{lll}
\mathbf{V}_{h}:=\mathbf{V} \cap\left[\mathbb{P}_{1}\left(\mathcal{T}_{h}\right)\right]^{d}, & Q_{h}:=Q \cap\left[C^{0}(\bar{\Omega}) \cap \mathbb{P}_{1}\left(\mathcal{T}_{H}\right)\right] & \text { on simplices, } \\
\mathbf{V}_{h}:=\mathbf{V} \cap\left[\mathbb{Q}_{1}\left(\mathcal{T}_{h}\right)\right]^{d}, & Q_{h}:=Q \cap\left[C^{0}(\bar{\Omega}) \cap \mathbb{Q}_{1}\left(\mathcal{T}_{H}\right)\right] & \text { on rect. par. }
\end{array}
$$

where $\mathcal{T}_{h}$ was formed from $\mathcal{T}_{H}$ by adding the edge barycenters as nodes and cutting each simplex into $2^{d}$ subsimplices and similarly for rectangular parallelepipeds.

\subsection{Stabilized schemes}

Let

$$
\begin{array}{ll}
\mathbf{V}_{h}:=\mathbf{V} \cap\left[\mathbb{P}_{l}\left(\mathcal{T}_{h}\right)\right]^{d}, & Q_{h}:=Q \cap\left[C^{0}(\bar{\Omega}) \cap \mathbb{P}_{l}\left(\mathcal{T}_{h}\right)\right] \quad \text { on simplices, } \\
\mathbf{V}_{h}:=\mathbf{V} \cap\left[\mathbb{Q}_{l}\left(\mathcal{T}_{h}\right)\right]^{d}, & Q_{h}:=Q \cap\left[C^{0}(\bar{\Omega}) \cap \mathbb{Q}_{l}\left(\mathcal{T}_{h}\right)\right] \quad \text { on rect. par., }
\end{array}
$$

and let $\delta>0$ be a penalization parameter. We in particular take into account the following methods with $t_{h}\left(\mathbf{u}_{h}, p_{h} ; \mathbf{v}_{h}\right):=0$ :

- The Brezzi-Pitkäranta method [18] on simplices, with $l=1$ and

$$
s_{h}\left(\mathbf{u}_{h}, p_{h} ; q_{h}\right):=-\delta \sum_{K \in \mathcal{T}_{h}} h_{K}^{2}\left(\nabla p_{h}, \nabla q_{h}\right)_{K} .
$$

- The Hughes-Franca-Balestra method [37, with $l \geq 1$ and

$$
s_{h}\left(\mathbf{u}_{h}, p_{h} ; q_{h}\right):=\delta \sum_{K \in \mathcal{T}_{h}} h_{K}^{2}\left(\mathbf{f}+\Delta \mathbf{u}_{h}-\nabla p_{h}, \nabla q_{h}\right)_{K} .
$$

- The Brezzi-Douglas method [16, with $l \geq 1$ and

$$
s_{h}\left(\mathbf{u}_{h}, p_{h} ; q_{h}\right):=\delta \sum_{K \in \mathcal{T}_{h}} h_{K}^{2}\left\{\left(\mathbf{f}-\nabla p_{h}, \nabla q_{h}\right)_{K}+\left\langle\Delta \mathbf{u}_{h} \cdot \mathbf{n}_{K}, q_{h}\right\rangle_{\partial K \cap \partial \Omega}\right\} .
$$

- The Dohrmann-Bochev method [23], with $l \geq 1$ and

$$
s_{h}\left(\mathbf{u}_{h}, p_{h} ; q_{h}\right):=-\left(p_{h}-\Pi_{l-1} p_{h}, q_{h}-\Pi_{l-1} q_{h}\right) ;
$$

here $\Pi_{l-1}$ is the $L^{2}(\Omega)$-orthogonal projection onto piecewise polynomials of total degree $l-1$ also when $\mathcal{T}_{h}$ consists of rectangular parallelepipeds. 
Many other other choices are also included in our analysis, in particular discontinuous pressure elements with no $C^{0}(\bar{\Omega})$ requirement for $Q_{h}$ such as

$$
\begin{array}{lll}
\mathbf{V}_{h}:=\mathbf{V} \cap\left[\mathbb{P}_{l}\left(\mathcal{T}_{h}\right)\right]^{d}, & Q_{h}:=Q \cap \mathbb{P}_{l-1}\left(\mathcal{T}_{h}\right) & \text { on simplices, } \\
\mathbf{V}_{h}:=\mathbf{V} \cap\left[\mathbb{Q}_{l}\left(\mathcal{T}_{h}\right)\right]^{d}, & Q_{h}:=Q \cap \mathbb{Q}_{l-1}\left(\mathcal{T}_{h}\right) & \text { on rect. par.: }
\end{array}
$$

- The Hughes-Franca 36] method, see also Franca and Stenberg 32, with a parameter $\rho=-1,1$, choices of the form $l \geq 1$ in 3.4 or $l \geq d$ on simplices and $l \geq 2$ in 3.5) on rectangular parallelepipeds, and

$$
\begin{aligned}
& s_{h}\left(\mathbf{u}_{h}, p_{h} ; q_{h}\right):=\delta \sum_{K \in \mathcal{T}_{h}} h_{K}^{2}\left(\mathbf{f}+\Delta \mathbf{u}_{h}-\nabla p_{h}, \nabla q_{h}\right)_{K}, \\
& t_{h}\left(\mathbf{u}_{h}, p_{h} ; \mathbf{v}_{h}\right):=\delta \rho \sum_{K \in \mathcal{T}_{h}} h_{K}^{2}\left(\mathbf{f}+\Delta \mathbf{u}_{h}-\nabla p_{h}, \Delta \mathbf{v}_{h}\right)_{K} ;
\end{aligned}
$$

note that the above stabilization $t_{h}(\cdot, \cdot ; \cdot)$ only contains the elementwise Laplacian of the test function, so that it indeed vanishes for the piecewise affine or $d$-affine test functions $\boldsymbol{\psi}_{\mathbf{a}, m}$.

- The Hughes-Franca [36]/Douglas-Wang [26] method, with $l=1$ in $[3.5], t_{h}\left(\mathbf{u}_{h}, p_{h} ; \mathbf{v}_{h}\right):=0$, and

$$
s_{h}\left(\mathbf{u}_{h}, p_{h} ; q_{h}\right):=-\delta \sum_{e \in \mathcal{E}_{h}^{\text {int }}} h_{e}\left(\llbracket p_{h} \rrbracket_{e}, \llbracket q_{h} \rrbracket_{e}\right)_{e} .
$$

- The Kechkar-Silvester locally stabilized method [40, with $l=1$ in 3.5$), t_{h}\left(\mathbf{u}_{h}, p_{h} ; \mathbf{v}_{h}\right):=0$, and

$$
s_{h}\left(\mathbf{u}_{h}, p_{h} ; q_{h}\right):=-\delta \sum_{K \in \mathcal{T}_{H}} \sum_{e \in \mathcal{E}_{h}^{\text {int }} ; e \subset K, e \not \subset \partial K} h_{e}\left(\llbracket p_{h} \rrbracket_{e}, \llbracket q_{h} \rrbracket_{e}\right)_{e},
$$

where $\mathcal{T}_{h}$ is a submesh of a macroelement partition $\mathcal{T}_{H}$; typically $\mathcal{T}_{H}$ consists of simplices/rectangular parallelepipeds and $\mathcal{T}_{h}$ is obtained by adding the edge barycenters as nodes and cutting each simplex into $2^{d}$ subsimplices and similarly for rectangular parallelepipeds.

- The Hughes-Franca [36]/Douglas-Wang [26]/Franca and Stenberg [32]/ Kechkar-Silvester [40] methods, combining the three above stabilizations, spaces, and macroelement partitions.

\section{Adaptive inexact iterative algorithms}

In this section, we introduce our adaptive inexact amendment of the iterative outer-inner loop Algorithm 1.3 as well as of the single loop Algoritm 1.4

Let $\eta_{\text {rem }}^{k, i}, \eta_{\text {alg, }}^{k, i}, \eta_{\text {alg, }, p}^{k, i}$, and $\eta_{\text {disc }}^{k, i}$ be respectively the estimators of the algebraic reminder, the inner algebraic (velocity) solver error, the outer (pressure) solver iteration error, and the discretization error on the $k$-th outer solver step and $i$-th algebraic step, see Corollary 6.2 below. We then modify Algorithm 1.3 as follows:

Algorithm 4.1 (Adaptive inexact outer-inner (Uzawa) algorithm).

1. In step 1. choose also a fixed additional iteration count $\nu_{0}>0$ and real parameters $\gamma_{\mathrm{rem}}, \gamma_{\mathrm{alg}, \mathbf{u}}$, $\gamma_{\mathrm{alg}, p}>0$, typically of order $10^{-1}$. Set $\nu:=\nu_{0}$.

2. In step 2(c)i, increase $i:=i+\nu_{0}$. 
3. In step 2(c)ii, on iteration $i$ of the inner solver for the approximation of 1.8, consider $\nu:=\nu_{0}$ additional iterations. This gives $\mathbf{u}_{h}^{k, i+\nu} \in \mathbf{V}_{h}$ and $\mathrm{R}^{k, i+\nu} \in \mathbb{R}^{M}$ satisfying

$$
\begin{array}{r}
\left(\nabla \mathbf{u}_{h}^{k, i+\nu}, \nabla \mathbf{v}_{j}\right)+t_{h}\left(\mathbf{u}_{h}^{k, i+\nu}, p_{h}^{k} ; \mathbf{v}_{j}\right)=\left(\nabla \cdot \mathbf{v}_{j}, p_{h}^{k}\right)+\left(\mathbf{f}, \mathbf{v}_{j}\right)-\mathrm{R}_{j}^{k, i+\nu} \\
1 \leq j \leq M
\end{array}
$$

Compute the estimators $\eta_{\mathrm{rem}}^{k, i}$ and $\eta_{\mathrm{alg}, \mathbf{u}}^{k, i}$. Check the balancing criterion

$$
\eta_{\mathrm{rem}}^{k, i} \leq \gamma_{\mathrm{rem}} \eta_{\mathrm{alg}, \mathbf{u}}^{k, i}
$$

If not satisfied, continue performing $\nu_{0}$ additional iterations and updating $\nu:=\nu+\nu_{0}$, until (4.2a) is satisfied.

4. Precise the inner algebraic solver stopping criterion 1.10 as: compute the estimators $\eta_{\mathrm{alg}, p}^{k, i}$ and $\eta_{\mathrm{disc}}^{k, i}$ and stop when

If not satisfied, update $i:=i+\nu$.

$$
\eta_{\mathrm{alg}, \mathbf{u}}^{k, i} \leq \gamma_{\mathrm{alg}, \mathbf{u}} \max \left\{\eta_{\mathrm{disc}}^{k, i}, \eta_{\mathrm{alg}, p}^{k, i}\right\}
$$

5. Precise the outer (Uzawa) stopping criterion 1.12 as:

$$
\eta_{\mathrm{alg}, p}^{k, i} \leq \gamma_{\mathrm{alg}, p} \eta_{\mathrm{disc}}^{k, i}
$$

Remark 4.2 (Local stopping criteria). Above, the stopping criteria 4.2 are global in the sense that the estimators are summed over all mesh elements. Alternatively, we can also consider the local stopping criteria [38, 29]:

$$
\begin{array}{rlrl}
\eta_{\mathrm{rem}, K}^{k, i} & \leq \gamma_{\mathrm{rem}, K} \eta_{\mathrm{alg}, \mathbf{u}, K}^{k, i}, & & \forall K \in \mathcal{T}_{h}, \\
\eta_{\mathrm{alg}, \mathbf{u}, K}^{k, i} \leq \gamma_{\mathrm{alg}, \mathbf{u}, K} \max \left\{\eta_{\mathrm{disc}, K}^{k, i}, \eta_{\mathrm{alg}, p, K}^{k,, i}\right\}, & & \forall K \in \mathcal{T}_{h}, \\
\eta_{\mathrm{alg}, p, K}^{k, i} \leq \gamma_{\mathrm{alg}, p, K} \eta_{\mathrm{disc}, K}^{k, i}, & & \forall K \in \mathcal{T}_{h},
\end{array}
$$

where for any $K \in \mathcal{T}_{h}$, the parameters $\gamma_{\mathrm{rem}, K}, \gamma_{\mathrm{alg}, \mathbf{u}, K}$, and $\gamma_{\mathrm{alg}, p, K}$ are positive user-given weights, typically of order $10^{-1}$.

Similarly, we propose to amend to the single-loop Algorithm 1.4

Algorithm 4.3 (Adaptive inexact single-loop algorithm).

1. In step 1, choose also a fixed additional iteration count $\nu_{0}>0$ and real parameters $\gamma_{\mathrm{rem}}$ and $\gamma_{\mathrm{alg}}>0$, typically of order $10^{-1}$. Set $\nu:=\nu_{0}$.

2. In step 2a, increase $i:=i+\nu_{0}$.

3. On the given iteration $i$ of the algebraic solver in step 2b, consider $\nu:=\nu_{0}$ additional iterations. This gives $\left(\mathbf{u}_{h}^{i+\nu}, p_{h}^{i+\nu}\right) \in \mathbf{V}_{h} \times Q_{h}$ with $\mathrm{R}_{\mathbf{u}}^{i+\nu} \in \mathbb{R}^{M}$ and $\mathrm{R}_{p}^{i+\nu} \in \mathbb{R}^{N}$ such that

$$
\begin{array}{rr}
\left(\nabla \mathbf{u}_{h}^{i+\nu}, \nabla \mathbf{v}_{j}\right)+t_{h}\left(\mathbf{u}_{h}^{i+\nu}, p_{h}^{i+\nu} ; \mathbf{v}_{j}\right)=\left(\nabla \cdot \mathbf{v}_{j}, p_{h}^{i+\nu}\right)+\left(\mathbf{f}, \mathbf{v}_{j}\right)-\mathrm{R}_{\mathbf{u}, j}^{i+\nu} \\
& \forall 1 \leq j \leq M, \\
-\left(\nabla \cdot \mathbf{u}_{h}^{i+\nu}, q_{j}\right)+s_{h}\left(\mathbf{u}_{h}^{i+\nu}, p_{h}^{i+\nu} ; q_{j}\right)=-\mathrm{R}_{p, j}^{i+\nu} & \\
& \forall 1 \leq j \leq N .
\end{array}
$$

Compute the estimators $\eta_{\mathrm{rem}}^{i}, \eta_{\mathrm{alg}, \mathbf{u}}^{i}$, and $\eta_{\mathrm{alg}, p}^{i}$. Check the balancing criterion

$$
\eta_{\mathrm{rem}}^{i} \leq \gamma_{\mathrm{rem}}\left(\eta_{\mathrm{alg}, \mathbf{u}}^{i}+\eta_{\mathrm{alg}, p}^{i}\right) .
$$

If not satisfied, continue performing $\nu_{0}$ additional iterations and updating $\nu:=\nu+\nu_{0}$, until 4.5a) is satisfied.

4. Precise the algebraic solver stopping criterion (1.14) as: compute the estimator $\eta_{\mathrm{disc}}^{i}$ and stop when

$$
\left(\eta_{\text {alg }, \mathbf{u}}^{i}+\eta_{\text {alg }, p}^{i}\right) \leq \gamma_{\text {alg }} \eta_{\text {disc }}^{i}
$$

If not satisfied, update $i:=i+\nu$.

Local stopping criteria as in Remark 4.2 can also be used here. 


\section{Construction of quasi-equilibrated stresses}

We show here how, under Assumption 3.1, quasi-equilibrated stresses can be obtained for the consider methods. In the context of inexact solvers, we follow essentially [29] and decompose the total stress into parts corresponding to the different error components. Henceforth, to abbreviate, $\left(\mathbf{u}_{h}^{k, i}, p_{h}^{k}\right)$ writes for the current approximation in Algorithm 4.1 (ex Algorithm 1.3), as well as for $\left(\mathbf{u}_{h}^{i}, p_{h}^{i}\right.$ ) in Algorithm 4.3 (ex Algorithm 1.4.

Recall the definition of the space $\mathbb{H}(\operatorname{div}, \Omega):=\left\{\mathbb{v} \in\left[L^{2}(\Omega)\right]^{d \times d} ; \nabla \cdot \mathbb{v} \in\left[L^{2}(\Omega)\right]^{d}\right\}$. For each vertex $\mathbf{a} \in \mathcal{V}_{h}$ and the associated patch $\omega_{\mathbf{a}}$, let $\mathbb{R} \mathbb{T N}_{l}\left(\omega_{\mathbf{a}}\right) \subset \mathbb{H}\left(\operatorname{div}, \omega_{\mathbf{a}}\right)$ be the Raviart-Thomas-Nédélec space of order $l \geq 0$ on $\omega_{\mathbf{a}}$. Each row of the functions therefrom needs to take the form $\left(\left[\mathbb{P}_{l}(K)\right]^{d}+\mathbb{P}_{l}(K) \mathbf{x}\right)^{t}$ on each simplex $K$ and $\mathbb{Q}_{l, l+1}(K) \times \mathbb{Q}_{l+1, l}(K)$ if $d=2$ and $\mathbb{Q}_{l, l+1, l+1}(K) \times \mathbb{Q}_{l+1, l, l+1}(K) \times \mathbb{Q}_{l+1, l+1, l}(K)$ if $d=3$ on each rectangular parallelepiped $K$. Let next $\mathbb{R T N}_{l}^{\mathrm{N}, 0}\left(\omega_{\mathbf{a}}\right)$ be the subspace of $\mathbb{R} \mathbb{T} \mathbb{N}_{l}\left(\omega_{\mathbf{a}}\right)$ with zero normal flux through $\partial \omega_{\mathbf{a}}$ for $\mathbf{a} \in \mathcal{V}_{h}^{\text {int }}$ and with zero normal flux through $\partial \omega_{\mathbf{a}} \backslash \partial \Omega$ for $\mathbf{a} \in \mathcal{V}_{h}^{\text {ext }}$. We will also need the space $\left[\mathbb{R}_{l}^{*}\left(\omega_{\mathbf{a}}\right)\right]^{d}$ spanned by piecewise polynomials on $\omega_{\mathbf{a}}$ of total degree at most $l$ on simplices and of degree at most $l$ in each variable for rectangular parallelepipeds. In addition, a zero mean value on $\omega_{\mathbf{a}}$ for each component is imposed when $\mathbf{a} \in \mathcal{V}_{h}^{\text {int }}$; the mean value condition is not imposed on $\mathbf{a} \in \mathcal{V}_{h}^{\text {ext }}$.

For each interior vertex $\mathbf{a} \in \mathcal{V}_{h}^{\text {int }}$ and each $1 \leq m \leq d$, define $\left(\mathrm{R}_{\mathbf{a}}^{k, i}\right)_{m}:=\mathrm{R}_{j}^{k, i}$ from 1.9 in Algorithm 4.1 and $\left(\mathrm{R}_{\mathbf{a}}^{k, i}\right)_{m}:=\mathrm{R}_{\mathbf{u}, j}^{i}$ from 1.13a in Algorithm 4.3, while taking the basis function $\mathbf{v}_{j}$ of $\mathbf{V}_{h}$ given by $\boldsymbol{\psi}_{\mathbf{a}, m}$. For notational convenience, set $\mathrm{R}_{\mathbf{a}}^{k, i}=\mathbf{0}$ for $\mathbf{a} \in \mathcal{V}_{h}^{\text {ext }}$. Following [21, 14, 29, 30, we reconstruct a Cauchy stress by solving local mixed finite element problems on the patches $\omega_{\mathbf{a}}$ :

Definition 5.1 (Discretization stress reconstruction $\left.\mathbb{d}_{h}^{k, i}\right)$. For each outer loop iteration $k \geq 0$, each inner algebraic solver iteration $i \geq 1$, and each patch $\omega_{\mathbf{a}}, \mathbf{a} \in \mathcal{V}_{h}$, construct $\left(\mathbb{d}_{\mathbf{a}}^{k, i}, \mathbf{o}_{\mathbf{a}}^{k, i}\right) \in \mathbb{R}_{\mathbb{N}} \mathbb{N}_{l}^{N, 0}\left(\omega_{\mathbf{a}}\right) \times\left[\mathbb{R}_{l}^{*}\left(\omega_{\mathbf{a}}\right)\right]^{d}$ such that

$$
\begin{aligned}
& \left(\mathbb{d}_{\mathbf{a}}^{k, i}, \mathbb{v}_{h}\right)_{\omega_{\mathbf{a}}}+\left(\mathbf{o}_{\mathbf{a}}^{k, i}, \nabla \cdot \mathbb{v}_{h}\right)_{\omega_{\mathbf{a}}}=\left(\left(\nabla \mathbf{u}_{h}^{k, i}-p_{h}^{k} \mathbb{I}\right) \psi_{\mathbf{a}}, \mathbb{v}_{h}\right)_{\omega_{\mathbf{a}}}, \quad \forall \mathbb{v}_{h} \in \mathbb{R T N}_{l}^{\mathrm{N}, 0}\left(\omega_{\mathbf{a}}\right), \\
& -\left(\left(\nabla \cdot \mathbb{d}_{\mathbf{a}}^{k, i}\right)_{m},\left(\mathbf{q}_{h}\right)_{m}\right)_{\omega_{\mathbf{a}}}=\left(\mathbf{f} \cdot \boldsymbol{\psi}_{\mathbf{a}, m}-\left(\nabla \mathbf{u}_{h}^{k, i}-p_{h}^{k} \mathbb{I}\right): \nabla \boldsymbol{\psi}_{\mathbf{a}, m},\left(\mathbf{q}_{h}\right)_{m}\right)_{\omega_{\mathbf{a}}} \\
& -\left(\left(\mathrm{R}_{\mathbf{a}}^{k, i}\right)_{m}\left|\omega_{\mathbf{a}}\right|^{-1},\left(\mathbf{q}_{h}\right)_{m}\right)_{\omega_{\mathbf{a}}}, \\
& \forall \mathbf{q}_{h} \in\left[\mathbb{R}_{l}^{*}\left(\omega_{\mathbf{a}}\right)\right]^{d}, 1 \leq m \leq d .
\end{aligned}
$$

Then set

$$
\mathbb{d}_{h}^{k, i}:=\sum_{\mathbf{a} \in \mathcal{V}_{h}} \mathbb{d}_{\mathbf{a}}^{k, i}
$$

We also need construct $\mathbb{d}_{h}^{k, i+\nu}$ by the same procedure, with merely $i+\nu$ in place of $i$.

Remark 5.2 (Neumann compatibility condition). Problems (5.1) are well-posed thanks to Assumption 3.1. Indeed, taking $\boldsymbol{\psi}_{\mathbf{a}, m} \in \mathbf{V}_{h}$ as the test functions respectively in (1.9) and (1.13a) implies

$$
\begin{array}{r}
\left(\mathbf{f}, \boldsymbol{\psi}_{\mathbf{a}, m}\right)_{\omega_{\mathbf{a}}}-\left(\nabla \mathbf{u}_{h}^{k, i}, \nabla \boldsymbol{\psi}_{\mathbf{a}, m}\right)_{\omega_{\mathbf{a}}}+\left(\nabla \cdot \boldsymbol{\psi}_{\mathbf{a}, m}, p_{h}^{k}\right)_{\omega_{\mathbf{a}}}-\left(\mathrm{R}_{\mathbf{a}}^{k, i}\right)_{m}=0 \\
\forall \mathbf{a} \in \mathcal{V}_{h}^{\text {int }}, 1 \leq m \leq d
\end{array}
$$

The same reasoning applies for $i+\nu$ in place of $i$, using 4.1. and 4.4a).

Remark 5.3 (Cheaper equilibration). Following [14], one can also carry the one-degree-lower reconstruction in $\mathbb{R}_{\mathbb{N}}^{N-1} \mathbb{N}_{l-1}\left(\omega_{\mathbf{a}}\right) \times\left[\mathbb{P}_{l-1}^{*}\left(\omega_{\mathbf{a}}\right)\right]^{d}$. Employing the Raviart-Thomas-Nédélec projector, the efficiency of such a simplification has been shown in [29], albeit the polynomial-degree-robustness has not been treated therein.

Lemma 5.4 (Quasi-equilibration). Let Assumption 3.1 hold. On step $i$ of the given algebraic solver applied to 1.8 or (1.7) and after $\nu>0$ additional iterations yielding 4.1) or 4.4a, construct $\mathbb{d}_{h}^{k, i+\nu}$ via Definition 5.1 and set

$$
\begin{aligned}
\left.\mathbf{r}_{h}^{k, i+\nu}\right|_{K} & :=\sum_{\mathbf{a} \in \mathcal{V}_{K}}\left|\omega_{\mathbf{a}}\right|^{-1} \mathrm{R}_{\mathbf{a}}^{k, i+\nu} \\
\mathbf{f}_{h} & :=\boldsymbol{\Pi}_{l} \mathbf{f} .
\end{aligned}
$$

Then

$$
-\nabla \cdot \mathbb{d}_{h}^{k, i+\nu}=\mathbf{f}_{h}-\mathbf{r}_{h}^{k, i+\nu} .
$$


Proof. Fix an arbitrary element $K \in \mathcal{T}_{h}$ and $\mathbf{q}_{h} \in\left[\mathbb{P}_{l}(K)\right]^{d}$ on simplices and $\mathbf{q}_{h} \in\left[\mathbb{Q}_{l}(K)\right]^{d}$ on rectangular parallelepipeds; $\mathbf{q}_{h}$ is only supported on the element $K$. For any vertex $\mathbf{a} \in \mathcal{V}_{K}$, take $\mathbf{q}_{h}$ as the test function in (5.1b) (this is possible thanks to the Neumann compatibility (5.3)). Then, for $1 \leq m \leq d$, we have

$$
\begin{aligned}
-\left(\left(\nabla \cdot \mathbb{d}_{h}^{k, i+\nu}\right)_{m},\left(\mathbf{q}_{h}\right)_{m}\right)_{K}= & -\sum_{\mathbf{a} \in \mathcal{V}_{K}}\left(\left(\nabla \cdot \mathbb{d}_{\mathbf{a}}^{k, i+\nu}\right)_{m},\left(\mathbf{q}_{h}\right)_{m}\right)_{K} \\
= & \sum_{\mathbf{a} \in \mathcal{V}_{K}}\left(\mathbf{f} \cdot \boldsymbol{\psi}_{\mathbf{a}, m}-\left(\nabla \mathbf{u}_{h}^{k, i+\nu}-p_{h}^{k} \mathbb{I}\right): \nabla \boldsymbol{\psi}_{\mathbf{a}, m},\left(\mathbf{q}_{h}\right)_{m}\right)_{K} \\
& -\left(\left(\mathrm{R}_{\mathbf{a}}^{k, i+\nu}\right)_{m}\left|\omega_{\mathbf{a}}\right|^{-1},\left(\mathbf{q}_{h}\right)_{m}\right)_{K} \\
= & \left(\left(\mathbf{f}_{h}-\mathbf{r}_{h}^{k, i+\nu}\right)_{m},\left(\mathbf{q}_{h}\right)_{m}\right)_{K},
\end{aligned}
$$

employing the partition of unity $\left.\sum_{\mathbf{a} \in \mathcal{V}_{K}} \boldsymbol{\psi}_{\mathbf{a}, m}\right|_{K}=1$ and $\left.\sum_{\mathbf{a} \in \mathcal{V}_{K}} \nabla \boldsymbol{\psi}_{\mathbf{a}, m}\right|_{K}=0$ together with the definitions (5.4).

Finally, following the idea in [38, Section 7.2] and [29, Section 4], see also the references therein, we identify a stress reconstruction that we use to estimate the algebraic error:

Definition 5.5 (Algebraic error stress reconstruction $\mathrm{a}_{h}^{k, i}$ ). Define the algebraic error stress reconstruction as

$$
\mathrm{a}_{h}^{k, i}:=\mathbb{d}_{h}^{k, i+\nu}-\mathbb{d}_{h}^{k, i},
$$

where $\mathbb{d}_{h}^{k, i}$ and $\mathbb{d}_{h}^{k, i+\nu}$ are constructed following Definition 5.1 .

Remark that $\left\|\mathrm{a}_{h}^{k, i}\right\| \rightarrow 0$ as the inner algebraic solver converges, which is a necessary condition for algebraic error control from [29, Section 3.2].

\section{Guaranteed reliability}

We now give a guaranteed estimate on the total error in particular valid on each iteration of Algorithms 4.1 or 4.3. We will use the Poincaré inequality

$$
\left\|\mathbf{v}-\mathbf{v}_{K}\right\|_{K} \leq \frac{h_{K}}{\pi}\|\nabla \mathbf{v}\|_{K}, \quad \forall \mathbf{v} \in\left[H^{1}(K)\right]^{d},
$$

where $\mathbf{v}_{K}$ denotes the mean value of $\mathbf{v}$ in $K$, see [46]. We will also employ the Friedrichs inequality stating that

$$
\|\mathbf{v}\| \leq h_{\Omega}\|\nabla \mathbf{v}\|, \quad \forall \mathbf{v} \in \mathbf{V} .
$$

Lemma 5.4 allows us to prove the following theorem, following essentially [25, 34, 29]. We for simplicity denote $\eta_{*}^{k, i}=\left\{\sum_{K \in \mathcal{T}_{h}}\left(\eta_{*, K}^{k, i}\right)^{2}\right\}^{1 / 2}$.

Theorem 6.1 (A guaranteed a posteriori estimate valid on each iteration). Let (u,p) be the weak solution of the Stokes problem of Definition 1.1 and let its finite element discretization of Definition 1.2 satisfy Assumption 3.1. Let $\left(\mathbf{u}_{h}^{k, i}, p_{h}^{k}\right) \in \mathbf{V}_{h} \times Q_{h}$ be the current approximation on arbitrary outer iteration $k \geq 0$ and inner algebraic iteration $i \geq 1$ in Algorithm 4.1 or 4.3. Consider $\nu>0$ additional algebraic iterations and construct $\mathbb{d}_{h}^{k, i+\nu}$ following Definition 5.1. For any $K \in \mathcal{T}_{h}$, define respectively the flux, divergence, algebraic remainder, and data oscillation estimators

$$
\begin{aligned}
\eta_{\mathrm{F}, K}^{k, i} & :=\left\|\nabla \mathbf{u}_{h}^{k, i}-p_{h}^{k} \mathbb{I}-\mathbb{d}_{h}^{k, i+\nu}\right\|_{K}, \\
\eta_{\mathrm{D}, K}^{k, i} & :=\beta^{-1}\left\|\nabla \cdot \mathbf{u}_{h}^{k, i}\right\|_{K}, \\
\eta_{\mathrm{rem}, K}^{k, i} & :=h_{\Omega}\left\|\mathbf{r}_{h}^{k, i+\nu}\right\|_{K}, \\
\eta_{\mathrm{osc}, K}^{k, i} & :=h_{K} \pi^{-1}\left\|\mathbf{f}-\mathbf{f}_{h}\right\|_{K} .
\end{aligned}
$$


Then we have

$$
\begin{array}{r}
\left\|\nabla\left(\mathbf{u}-\mathbf{u}_{h}^{k, i}\right)\right\| \leq \eta_{\mathrm{F}}^{k, i}+\eta_{\mathrm{D}}^{k, i}+\eta_{\mathrm{rem}}^{k, i}+\eta_{\mathrm{osc}}^{k, i} \\
\beta\left\|p-p_{h}^{k}\right\| \leq \eta_{\mathrm{F}}^{k, i}+\eta_{\mathrm{D}}^{k, i}+\eta_{\mathrm{rem}}^{k, i}+\eta_{\mathrm{osc}}^{k, i}
\end{array}
$$

Proof. We first bound $\left\|\nabla\left(\mathbf{u}-\mathbf{u}_{h}^{k, i}\right)\right\|$. Let $\mathbf{s} \in \mathbf{V}$ with $\nabla \cdot \mathbf{s}=0$ be the solution of

$$
(\nabla \mathbf{s}, \nabla \mathbf{v})=\left(\nabla \mathbf{u}_{h}^{k, i}, \nabla \mathbf{v}\right) \quad \forall \mathbf{v} \in \mathbf{V} \text { with } \nabla \cdot \mathbf{v}=0 .
$$

This problem has a unique solution, similarly to that of 1.5 . We have the Pythagorean equality

$$
\left\|\nabla\left(\mathbf{u}-\mathbf{u}_{h}^{k, i}\right)\right\|^{2}=\|\nabla(\mathbf{u}-\mathbf{s})\|^{2}+\left\|\nabla\left(\mathbf{s}-\mathbf{u}_{h}^{k, i}\right)\right\|^{2},
$$

which follows from the fact that

$$
\begin{aligned}
\left\|\nabla\left(\mathbf{u}-\mathbf{u}_{h}^{k, i}\right)\right\|^{2} & =\left\|\nabla\left(\mathbf{u}-\mathbf{s}+\mathbf{s}-\mathbf{u}_{h}^{k, i}\right)\right\|^{2} \\
& =\|\nabla(\mathbf{u}-\mathbf{s})\|^{2}+\left\|\nabla\left(\mathbf{s}-\mathbf{u}_{h}^{k, i}\right)\right\|^{2}+2\left(\nabla(\mathbf{u}-\mathbf{s}), \nabla\left(\mathbf{s}-\mathbf{u}_{h}^{k, i}\right)\right),
\end{aligned}
$$

with the last term disappearing thanks the facts that $\mathbf{u}-\mathbf{s} \in \mathbf{V}$ and $\nabla \cdot(\mathbf{u}-\mathbf{s})=0$ and to $(6.5)$. We estimate the two terms in 6.6 separately.

Using the expression of the energy norm as a dual norm, we have

$$
\|\nabla(\mathbf{u}-\mathbf{s})\|=\sup _{\boldsymbol{\varphi} \in \mathbf{V} ; \nabla \cdot \boldsymbol{\varphi}=0,\|\nabla \boldsymbol{\varphi}\|=1}(\nabla(\mathbf{u}-\mathbf{s}), \nabla \boldsymbol{\varphi})
$$

Let thus $\varphi \in \mathbf{V}$ with $\nabla \cdot \varphi=0$ and $\|\nabla \varphi\|=1$ be fixed. Employing the definitions (1.5) and (6.5), we have

$$
(\nabla(\mathbf{u}-\mathbf{s}), \nabla \varphi)=(\mathbf{f}, \boldsymbol{\varphi})-\left(\nabla \mathbf{u}_{h}^{k, i}, \nabla \varphi\right)
$$

Next, using that $0=\left(p_{h}^{k}, \nabla \cdot \boldsymbol{\varphi}\right)=\left(p_{h}^{k} \mathbb{I}, \nabla \boldsymbol{\varphi}\right)$, adding and subtracting $\left(\mathbb{d}_{h}^{k, i+\nu}, \nabla \boldsymbol{\varphi}\right)$, and using the Green theorem, we get

$$
(\nabla(\mathbf{u}-\mathbf{s}), \nabla \boldsymbol{\varphi})=\left(\mathbf{f}+\nabla \cdot \mathbb{d}_{h}^{k, i+\nu}, \boldsymbol{\varphi}\right)-\left(\nabla \mathbf{u}_{h}^{k, i}-p_{h}^{k} \mathbb{I}-\mathbb{d}_{h}^{k, i+\nu}, \nabla \boldsymbol{\varphi}\right)
$$

Using (5.5), we further infer

$$
(\nabla(\mathbf{u}-\mathbf{s}), \nabla \boldsymbol{\varphi})=\left(\mathbf{f}-\mathbf{f}_{h}, \boldsymbol{\varphi}\right)+\left(\mathbf{r}_{h}^{k, i+\nu}, \boldsymbol{\varphi}\right)-\left(\nabla \mathbf{u}_{h}^{k, i}-p_{h}^{k} \mathbb{I}-\mathbb{d}_{h}^{k, i+\nu}, \nabla \boldsymbol{\varphi}\right) .
$$

We have, for any $K \in \mathcal{T}_{h}$,

$$
\left(\mathbf{f}-\mathbf{f}_{h}, \boldsymbol{\varphi}\right)_{K}=\left(\mathbf{f}-\mathbf{f}_{h}, \boldsymbol{\varphi}-\boldsymbol{\varphi}_{K}\right)_{K} \leq \eta_{\mathrm{osc}, K}^{k, i}\|\nabla \boldsymbol{\varphi}\|_{K},
$$

using the Poincaré inequality 6.1, whereas the estimate

$$
\left(\nabla \mathbf{u}_{h}^{k, i}-p_{h}^{k} \mathbb{I}-\mathbb{d}_{h}^{k, i+\nu}, \nabla \varphi\right)_{K} \leq \eta_{\mathrm{F}, K}^{k, i}\|\nabla \varphi\|_{K}
$$

follows immediately by the Cauchy-Schwarz inequality. With the Friedrichs inequality (6.2), we obtain

$$
\left(\mathbf{r}_{h}^{k, i+\nu}, \boldsymbol{\varphi}\right) \leq h_{\Omega}\left\|\mathbf{r}_{h}^{k, i+\nu}\right\|\|\nabla \boldsymbol{\varphi}\| .
$$

Thus the Cauchy-Schwarz inequality finally gives

$$
\begin{aligned}
(\nabla(\mathbf{u}-\mathbf{s}), \nabla \boldsymbol{\varphi}) & \leq \sum_{K \in \mathcal{T}_{h}}\left\{\left(\eta_{\mathrm{F}, K}^{k, i}+\eta_{\mathrm{osc}, K}^{k, i}\right)\|\nabla \boldsymbol{\varphi}\|_{K}\right\}+h_{\Omega}\left\|\mathbf{r}_{h}^{k, i+\nu}\right\|\|\nabla \boldsymbol{\varphi}\| \\
& \leq \eta_{\mathrm{F}}^{k, i}+\eta_{\mathrm{osc}}^{k, i}+\eta_{\mathrm{rem}}^{k, i} .
\end{aligned}
$$


We now treat the term $\left\|\nabla\left(\mathbf{s}-\mathbf{u}_{h}^{k, i}\right)\right\|$. We have

$$
\left\|\nabla\left(\mathbf{s}-\mathbf{u}_{h}^{k, i}\right)\right\|^{2}=\left(\nabla\left(\mathbf{s}-\mathbf{u}_{h}^{k, i}\right), \nabla\left(\mathbf{s}-\mathbf{u}_{h}^{k, i}\right)\right) .
$$

As in 1.5 with respect to 1.3 , the following equivalent formulation of 6.5 can be given: find $(\mathbf{s}, w) \in$ $\mathbf{V} \times Q$ such that

$$
\begin{aligned}
(\nabla \mathbf{s}, \nabla \mathbf{v})-(\nabla \cdot \mathbf{v}, w) & =\left(\nabla \mathbf{u}_{h}^{k, i}, \nabla \mathbf{v}\right) & & \forall \mathbf{v} \in \mathbf{V}, \\
-(\nabla \cdot \mathbf{s}, q) & =0 & & \forall q \in Q .
\end{aligned}
$$

Thus we have, as $\mathbf{s}-\mathbf{u}_{h}^{k, i} \in \mathbf{V}$ can be taken as a test function in (6.9a),

$$
\left(\nabla\left(\mathbf{s}-\mathbf{u}_{h}^{k, i}\right), \nabla\left(\mathbf{s}-\mathbf{u}_{h}^{k, i}\right)\right)=\left(\nabla \cdot\left(\mathbf{s}-\mathbf{u}_{h}^{k, i}\right), w\right)=-\left(\nabla \cdot \mathbf{u}_{h}^{k, i}, w\right) \leq\left\|\nabla \cdot \mathbf{u}_{h}^{k, i}\right\|\|w\| .
$$

We have also used the fact that $\nabla \cdot \mathbf{s}=0$ and the Cauchy-Schwarz inequality. To estimate $\|w\|$, we will rely on the inf-sup condition 1.4:

$$
\|w\| \leq \frac{1}{\beta} \sup _{\mathbf{v} \in \mathbf{V}} \frac{(w, \nabla \cdot \mathbf{v})}{\|\nabla \mathbf{v}\|}=\frac{1}{\beta} \sup _{\mathbf{v} \in \mathbf{V}} \frac{\left(\nabla\left(\mathbf{s}-\mathbf{u}_{h}^{k, i}\right), \nabla \mathbf{v}\right)}{\|\nabla \mathbf{v}\|} \leq \frac{1}{\beta}\left\|\nabla\left(\mathbf{s}-\mathbf{u}_{h}^{k, i}\right)\right\|,
$$

where we have employed (6.9a) and the Cauchy-Schwarz inequality. We thus reveal

$$
\left(\nabla\left(\mathbf{s}-\mathbf{u}_{h}^{k, i}\right), \nabla\left(\mathbf{s}-\mathbf{u}_{h}^{k, i}\right)\right) \leq \frac{\left\|\nabla \cdot \mathbf{u}_{h}^{k, i}\right\|}{\beta}\left\|\nabla\left(\mathbf{s}-\mathbf{u}_{h}^{k, i}\right)\right\|
$$

whence

$$
\left\|\nabla\left(\mathbf{s}-\mathbf{u}_{h}^{k, i}\right)\right\| \leq \eta_{\mathrm{D}}^{k, i} .
$$

We are left to treat the term $\left\|p-p_{h}^{k}\right\|$. We do so through the inf-sup condition (1.4), which in particular gives

$$
\left\|p-p_{h}^{k}\right\| \leq \frac{1}{\beta} \sup _{\boldsymbol{\varphi} \in \mathbf{V} ;\|\nabla \boldsymbol{\varphi}\|=1}\left(p-p_{h}^{k}, \nabla \cdot \boldsymbol{\varphi}\right) .
$$

Fix $\varphi \in \mathbf{V}$ with $\|\nabla \varphi\|=1$. The weak solution characterization 1.3a) gives

$$
(p, \nabla \cdot \boldsymbol{\varphi})=(\nabla \mathbf{u}, \nabla \boldsymbol{\varphi})-(\mathbf{f}, \boldsymbol{\varphi}) .
$$

Thus using also $\left(p_{h}^{k}, \nabla \cdot \varphi\right)=\left(p_{h}^{k} \mathbb{I}, \nabla \boldsymbol{\varphi}\right)$, adding and subtracting $\left(\mathbb{d}_{h}^{k, i+\nu}, \nabla \boldsymbol{\varphi}\right)$ as well as $\left(\nabla \mathbf{u}_{h}^{k, i}, \nabla \boldsymbol{\varphi}\right)$, and using the Green theorem, we arrive at

$$
\begin{aligned}
\left(p-p_{h}^{k}, \nabla \cdot \boldsymbol{\varphi}\right)= & \left(\nabla\left(\mathbf{u}-\mathbf{u}_{h}^{k, i}\right), \nabla \boldsymbol{\varphi}\right)-\left(\nabla \cdot \mathbb{d}_{h}^{k, i+\nu}+\mathbf{f}, \boldsymbol{\varphi}\right) \\
& +\left(\nabla \mathbf{u}_{h}^{k, i}-p_{h}^{k} \mathbb{I}-\mathbb{d}_{h}^{k, i+\nu}, \nabla \boldsymbol{\varphi}\right) .
\end{aligned}
$$

The two last terms on the above right-hand side could be estimated as in $(6.8)$ and the first one could be bounded by $\left\|\nabla\left(\mathbf{u}-\mathbf{u}_{h}^{k, i}\right)\right\|$ and consequently by 6.4a). Such a straightforward bound can, however, be substantially improved while proceeding as in reference [1]. Let $\varphi_{\mathrm{C}} \in \mathrm{V}$ with $\nabla \cdot \varphi_{\mathrm{C}}=0$ be the solution of

$$
\left(\nabla \varphi_{\mathrm{C}}, \nabla \mathbf{v}\right)=(\nabla \varphi, \nabla \mathbf{v}) \quad \forall \mathbf{v} \in \mathbf{V} \text { with } \nabla \cdot \mathbf{v}=0 .
$$

Let $\varphi_{\mathrm{NC}}:=\varphi-\varphi_{\mathrm{C}}$, and note that

$$
\left(\nabla \varphi_{\mathrm{NC}}, \nabla \mathbf{v}\right)=0 \quad \forall \mathbf{v} \in \mathbf{V} \text { with } \nabla \cdot \mathbf{v}=0 .
$$

Then, as in (6.6), we immediately have

$$
\|\nabla \varphi\|^{2}=\left\|\nabla \varphi_{\mathrm{C}}\right\|^{2}+\left\|\nabla \varphi_{\mathrm{NC}}\right\|^{2},
$$

as $\left(\nabla \varphi_{\mathrm{NC}}, \nabla \varphi_{\mathrm{C}}\right)=0$. Now

$$
\left(p-p_{h}^{k}, \nabla \cdot \boldsymbol{\varphi}\right)=\left(p-p_{h}^{k}, \nabla \cdot \boldsymbol{\varphi}_{\mathrm{NC}}\right)
$$


and

$$
\begin{aligned}
\left(p-p_{h}^{k}, \nabla \cdot \boldsymbol{\varphi}_{\mathrm{NC}}\right)= & \left(\nabla\left(\mathbf{u}-\mathbf{u}_{h}^{k, i}\right), \nabla \boldsymbol{\varphi}_{\mathrm{NC}}\right)-\left(\nabla \cdot \mathbb{d}_{h}^{k, i+\nu}+\mathbf{f}, \boldsymbol{\varphi}_{\mathrm{NC}}\right) \\
& +\left(\nabla \mathbf{u}_{h}^{k, i}-p_{h}^{k} \mathbb{I}-\mathbb{d}_{h}^{k, i+\nu}, \nabla \boldsymbol{\varphi}_{\mathrm{NC}}\right) .
\end{aligned}
$$

We can estimate

$$
-\left(\nabla \cdot \mathbb{d}_{h}^{k, i+\nu}+\mathbf{f}, \varphi_{\mathrm{NC}}\right)+\left(\nabla \mathbf{u}_{h}^{k, i}-p_{h}^{k} \mathbb{I}-\mathbb{d}_{h}^{k, i+\nu}, \nabla \varphi_{\mathrm{NC}}\right) \leq \eta_{\mathrm{F}}^{k, i}+\eta_{\mathrm{osc}}^{k, i}+\eta_{\mathrm{rem}}^{k, i},
$$

as in $(6.8)$, since $\left\|\nabla \varphi_{\mathrm{NC}}\right\| \leq\|\nabla \varphi\|=1$ by 6.12 . Finally for the term $\left(\nabla\left(\mathbf{u}-\mathbf{u}_{h}^{k, i}\right), \nabla \varphi_{\mathrm{NC}}\right)$, we have

$$
\left(\nabla\left(\mathbf{u}-\mathbf{u}_{h}^{k, i}\right), \nabla \varphi_{\mathrm{NC}}\right)=\left(\nabla\left(\mathbf{s}-\mathbf{u}_{h}^{k, i}\right), \nabla \varphi_{\mathrm{NC}}\right),
$$

as $\left(\nabla(\mathbf{u}-\mathbf{s}), \nabla \varphi_{\mathrm{NC}}\right)=0$ by $(6.11)$ since $\mathbf{u}-\mathbf{s} \in \mathbf{V}$ and $\nabla \cdot(\mathbf{u}-\mathbf{s})=0$. Now the Cauchy-Schwarz inequality and (6.10) together with $\left\|\nabla \varphi_{\mathrm{NC}}\right\| \leq\|\nabla \varphi\|=1$ give

$$
\left(\nabla\left(\mathbf{u}-\mathbf{u}_{h}^{k, i}\right), \nabla \varphi_{\mathrm{NC}}\right) \leq \eta_{\mathrm{D}}^{k, i} .
$$

Combining the above results gives 6.4b).

The algebraic error stress reconstruction $a_{h}^{k, i}$ of Definition 5.5 is following [29, Section 4] together with the algebraic remainder term $\eta_{\mathrm{rem}}^{k, i}$ given by $(6.3 \mathrm{c})$ designed to control the algebraic error in the velocity equation (1.6a). Let $\delta_{h}^{k, i} \in Q_{h}$ be given by (1.11). Note that it follows from (1.6b) that upon the convergence of any iterative solver, $\delta_{h}^{k, i}=0$. Our developments suggest to use $\beta^{-1}\left\|\delta_{h}^{k, i}\right\|$ for the estimate of the algebraic error in the pressure equation 1.6b). This yields altogether (as always, disregard the outer iteration index $k$ for the single-loop Algorithm 4.3 ):

Corollary 6.2 (A posteriori estimate distinguishing error components). Let the assumptions of Theorem 6.1 be satisfied. Then

$$
\left\|\nabla\left(\mathbf{u}-\mathbf{u}_{h}^{k, i}\right)\right\|+\beta\left\|p-p_{h}^{k}\right\| \leq 2\left(\eta_{\text {disc }}^{k, i}+\eta_{\text {alg, }}^{k, i}+\eta_{\text {alg }, p}^{k, i}+\eta_{\mathrm{rem}}^{k, i}+\eta_{\mathrm{osc}}^{k, i}\right),
$$

with $\eta_{\mathrm{rem}}^{k, i}$ and $\eta_{\mathrm{osc}}^{k, i}$ given respectively by $(6.3 \mathrm{c})$ and $(6.3 \mathrm{~d})$, and with the discretization, velocity equation algebraic error, and pressure equation algebraic error estimators respectively given by

$$
\begin{aligned}
\eta_{\text {disc }, K}^{k, i} & :=\left\|\nabla \mathbf{u}_{h}^{k, i}-p_{h}^{k} \mathbb{I}-\mathbb{d}_{h}^{k, i}\right\|_{K}+\beta^{-1}\left\|\nabla \cdot \mathbf{u}_{h}^{k, i}-\delta_{h}^{k, i}\right\|_{K}, \\
\eta_{\text {alg,u, }, K}^{k, i} & :=\left\|\mathrm{a}_{h}^{k, i}\right\|_{K}, \\
\eta_{\text {alg }, p, K}^{k, i} & :=\beta^{-1}\left\|\delta_{h}^{k, i}\right\|_{K} .
\end{aligned}
$$

Proof. The decomposition (5.6) and the triangle inequality yield

$$
\left\|\nabla \mathbf{u}_{h}^{k, i}-p_{h}^{k} \mathbb{I}-\mathbb{d}_{h}^{k, i+\nu}\right\|_{K} \leq\left\|\nabla \mathbf{u}_{h}^{k, i}-p_{h}^{k} \mathbb{I}-\mathbb{d}_{h}^{k, i}\right\|_{K}+\left\|\mathrm{a}_{h}^{k, i}\right\|_{K} .
$$

Another triangle inequality leads to

$$
\beta^{-1}\left\|\nabla \cdot \mathbf{u}_{h}^{k, i}\right\| \leq \beta^{-1}\left\|\nabla \cdot \mathbf{u}_{h}^{k, i}-\delta_{h}^{k, i}\right\|+\beta^{-1}\left\|\delta_{h}^{k, i}\right\| .
$$

In these two inequalities, the first terms contribute to the discretization error, whereas the second ones form the two components of the algebraic error.

\section{Polynomial-degree-robust (local) efficiency}

In this section, we prove the polynomial-degree-robust efficiency of the a posteriori error estimate of Corollary 6.2. We extend thereby the work [14 for the Laplace equation on triangles or rectangular parallelepipeds (see also [30]) and [31] for the Laplace equation on tetrahedra to the Stokes setting. To treat inexact solvers, we proceed as in [29]. For the inner-outer loop Algorithm 4.1, there in particular holds: 
Theorem 7.1 (Polynomial-degree-robust local efficiency). Let the assumptions of Theorem 6.1 be satisfied, together with the local stopping criteria 4.3 of Remark 4.2. Suppose that $0<\gamma_{\mathrm{rem}, K}, \gamma_{\mathrm{alg}, \mathbf{u}, K} \leq 1$ and that $0<\gamma_{\mathrm{alg}, p, K}<1$. Then, with the constants $C_{\mathrm{st}}$ and $C_{\mathrm{PF}, \omega_{\mathbf{a}}}>0$ only depending on the shape regularity parameter $\kappa_{\mathcal{T}}$, see [30, 31], there holds, for all $K \in \mathcal{T}_{h}$,

$$
\begin{aligned}
& \eta_{\mathrm{disc}, K}^{k, i}+\eta_{\mathrm{alg}, \mathbf{u}, K}^{k, i}+\eta_{\mathrm{alg}, p, K}^{k, i}+\eta_{\mathrm{rem}, K}^{k, i} \\
\leq & 4\left(1+\gamma_{\mathrm{alg}, p, K}\left(1-\gamma_{\mathrm{alg}, p, K}\right)^{-1}\right) C_{\mathrm{st}} \sum_{m=1}^{d} \sum_{\mathbf{a} \in \mathcal{V}_{K}} \max \left\{1, \beta^{-1}\right\} \\
& \times\left(2 C_{\mathrm{PF}, \omega_{\mathbf{a}}} h_{\omega_{\mathbf{a}}}\left\|\nabla \psi_{\mathbf{a}, m}\right\|_{\omega_{\mathbf{a}}, \infty}+1\right)\left(\left\|\nabla\left(\mathbf{u}-\mathbf{u}_{h}^{k, i}\right)\right\|_{\omega_{\mathbf{a}}}+\beta\left\|p-p_{h}^{k}\right\|_{\omega_{\mathbf{a}}}\right) \\
& +4\left(1-\gamma_{\mathrm{alg}, p, K}\right)^{-1} \beta^{-1}\left\|\nabla\left(\mathbf{u}-\mathbf{u}_{h}^{k, i}\right)\right\|_{K}+4\left(1+\gamma_{\mathrm{alg}, p, K}\left(1-\gamma_{\mathrm{alg}, p, K}\right)^{-1}\right) C_{\mathrm{st}} \\
& \times \sum_{m=1}^{d} \sum_{\mathbf{a} \in \mathcal{V}_{K}}\left\{\sum_{K^{\prime} \in \omega_{\mathbf{a}}}\left(\frac{h_{K^{\prime}}}{\pi}\left\|\mathbf{\Pi}_{l}\left(\mathbf{f} \cdot \boldsymbol{\psi}_{\mathbf{a}, m}\right)-\mathbf{f} \cdot \boldsymbol{\psi}_{\mathbf{a}, m}\right\|_{K^{\prime}}\right)^{2}\right\}^{1 / 2} .
\end{aligned}
$$

Proof. From our local stopping criteria 4.3, we have

$$
\eta_{\mathrm{rem}, K}^{k, i}+\eta_{\mathrm{alg}, \mathbf{u}, K}^{k, i}+\eta_{\mathrm{alg}, p, K}^{k, i} \leq 3 \eta_{\mathrm{disc}, K}^{k, i}
$$

Then, by 6.14a), we only need to bound $\left\|\nabla \mathbf{u}_{h}^{k, i}-p_{h}^{k} \mathbb{I}-\mathbb{d}_{h}^{k, i}\right\|_{K}$ and $\beta^{-1}\left\|\nabla \cdot \mathbf{u}_{h}^{k, i}-\delta_{h}^{k, i}\right\|_{K}$.

Using the triangle inequality, the definition 6.14c, the divergence-free property of the exact solution $\nabla \cdot \mathbf{u}=0$, and the local stopping criterion 4.3c), we obtain

$$
\begin{aligned}
\beta^{-1}\left\|\nabla \cdot \mathbf{u}_{h}^{k, i}-\delta_{h}^{k, i}\right\|_{K} & \leq \beta^{-1}\left\|\nabla \cdot \mathbf{u}_{h}^{k, i}\right\|_{K}+\beta^{-1}\left\|\delta_{h}^{k, i}\right\|_{K} \\
& \leq \beta^{-1}\left\|\nabla \cdot\left(\mathbf{u}-\mathbf{u}_{h}^{k, i}\right)\right\|_{K}+\gamma_{\text {alg }, p, K} \eta_{\text {disc }, K}^{k, i}
\end{aligned}
$$

Then the fact $\left\|\nabla \cdot\left(\mathbf{u}-\mathbf{u}_{h}^{k, i}\right)\right\| \leq\left\|\nabla\left(\mathbf{u}-\mathbf{u}_{h}^{k, i}\right)\right\|$ and the definition 6.14a yield

$$
\begin{aligned}
& \left(1-\gamma_{\mathrm{alg}, p, K}\right) \beta^{-1}\left\|\nabla \cdot \mathbf{u}_{h}^{k, i}-\delta_{h}^{k, i}\right\|_{K} \\
\leq & \beta^{-1}\left\|\nabla\left(\mathbf{u}-\mathbf{u}_{h}^{k, i}\right)\right\|_{K}+\gamma_{\mathrm{alg}, p, K}\left\|\nabla \mathbf{u}_{h}^{k, i}-p_{h}^{k} \mathbb{I}-\mathbb{d}_{h}^{k, i}\right\|_{K},
\end{aligned}
$$

where we also use the assumption $\gamma_{\mathrm{alg}, p, K}<1$.

To bound $\left\|\nabla \mathbf{u}_{h}^{k, i}-p_{h}^{k} \mathbb{I}-\mathbb{d}_{h}^{k, i}\right\|_{K}$, we use the same augments as in [29, 30] and proceed in three steps.

Step 1: Let $\mathbf{a} \in \mathcal{V}_{K}$ and consider the patch $\omega_{\mathbf{a}}$ of the vertex a. Define the function space $H_{*}^{1}\left(\omega_{\mathbf{a}}\right)$

$$
\begin{array}{ll}
H_{*}^{1}\left(\omega_{\mathbf{a}}\right)=\left\{v \in H^{1}\left(\omega_{\mathbf{a}}\right),(v, 1)_{\omega_{\mathbf{a}}}=0\right\}, & \mathbf{a} \in \mathcal{V}_{h}^{\text {int }}, \\
H_{*}^{1}\left(\omega_{\mathbf{a}}\right)=\left\{v \in H^{1}\left(\omega_{\mathbf{a}}\right), v=0 \text { on } \partial \omega_{\mathbf{a}} \cap \partial \Omega\right\}, & \mathbf{a} \in \mathcal{V}_{h}^{\text {ext }} .
\end{array}
$$

Let $1 \leq m \leq d$ and define $r_{\mathbf{a}, m} \in H_{*}^{1}\left(\omega_{\mathbf{a}}\right)$ such that for all $v \in H_{*}^{1}\left(\omega_{\mathbf{a}}\right)$,

$$
\begin{aligned}
\left(\nabla r_{\mathbf{a}, m}, \nabla v\right)_{\omega_{\mathbf{a}}}= & -\left(\left(\nabla \mathbf{u}_{h}^{k, i}-p_{h}^{k} \mathbb{I}\right) \boldsymbol{\psi}_{\mathbf{a}, m}, \nabla v\right)_{\omega_{\mathbf{a}}} \\
& +\left(\mathbf{f} \cdot \boldsymbol{\psi}_{\mathbf{a}, m}-\left(\nabla \mathbf{u}_{h}^{k, i}-p_{h}^{k} \mathbb{I}\right): \nabla \boldsymbol{\psi}_{\mathbf{a}, m}-\left(\mathrm{R}_{\mathbf{a}}^{k, i}\right)_{m}\left|\omega_{\mathbf{a}}\right|^{-1}, v\right)_{\omega_{\mathbf{a}}}
\end{aligned}
$$

We now prove that

$$
\begin{aligned}
\left\|\nabla r_{\mathbf{a}, m}\right\|_{\omega_{\mathbf{a}}} & \leq \max \left\{1, \beta^{-1}\right\}\left(2 C_{\mathrm{PF}, \omega_{\mathbf{a}}} h_{\omega_{\mathbf{a}}}\left\|\nabla \psi_{\mathbf{a}, m}\right\|_{\left.\omega_{\mathbf{a}, \infty}+1\right)}\right. \\
& \times\left(\left\|\nabla\left(\mathbf{u}-\mathbf{u}_{h}^{k, i}\right)\right\|_{\omega_{\mathbf{a}}}+\beta\left\|p-p_{h}^{k}\right\|_{\omega_{\mathbf{a}}}\right) .
\end{aligned}
$$

We have

$$
\left\|\nabla r_{\mathbf{a}, m}\right\|_{\omega_{\mathbf{a}}}=\sup _{v \in H_{*}^{1}\left(\omega_{\mathbf{a}}\right),\|\nabla v\|_{\omega_{\mathbf{a}}}=1}\left(\nabla r_{\mathbf{a}, m}, \nabla v\right)_{\omega_{\mathbf{a}}}
$$


Fix $v \in H_{*}^{1}\left(\omega_{\mathbf{a}}\right)$ with $\|\nabla v\|_{\omega_{\mathbf{a}}}=1$; then $(7.1)$ gives

$$
\begin{aligned}
& \left(\nabla r_{\mathbf{a}, m}, \nabla v\right)_{\omega_{\mathbf{a}}} \\
= & -\left(\left(\nabla \mathbf{u}_{h}^{k, i}-p_{h}^{k} \mathbb{I}\right) \boldsymbol{\psi}_{\mathbf{a}, m}, \nabla v\right)_{\omega_{\mathbf{a}}} \\
& +\left(\mathbf{f} \cdot \boldsymbol{\psi}_{\mathbf{a}, m}-\left(\nabla \mathbf{u}_{h}^{k, i}-p_{h}^{k} \mathbb{I}\right): \nabla \boldsymbol{\psi}_{\mathbf{a}, m}-\left(\mathrm{R}_{\mathbf{a}}^{k, i}\right)_{m}\left|\omega_{\mathbf{a}}\right|^{-1}, v\right)_{\omega_{\mathbf{a}}} \\
= & \left.\left(\mathbf{f} \cdot \boldsymbol{\psi}_{\mathbf{a}, m}, v\right)_{\omega_{\mathbf{a}}}-\left(\nabla \mathbf{u}_{h}^{k, i}-p_{h}^{k} \mathbb{I}\right), \nabla\left(\boldsymbol{\psi}_{\mathbf{a}, m} v\right)\right)_{\omega_{\mathbf{a}}}-\left(\left(\mathrm{R}_{\mathbf{a}}^{k, i}\right)_{m}\left|\omega_{\mathbf{a}}\right|^{-1}, v\right)_{\omega_{\mathbf{a}}} \\
= & \left.\left((\nabla \mathbf{u}-p \mathbb{I}), \nabla\left(\boldsymbol{\psi}_{\mathbf{a}, m} v\right)\right)_{\omega_{\mathbf{a}}}-\left(\nabla \mathbf{u}_{h}^{k, i}-p_{h}^{k} \mathbb{I}\right), \nabla\left(\boldsymbol{\psi}_{\mathbf{a}, m} v\right)\right)_{\omega_{\mathbf{a}}} \\
& -\left(\left(\mathrm{R}_{\mathbf{a}}^{k, i}\right)_{m}\left|\omega_{\mathbf{a}}\right|^{-1}, v\right)_{\omega_{\mathbf{a}}} \\
\leq & \left(\left\|\nabla\left(\mathbf{u}-\mathbf{u}_{h}^{k, i}\right)\right\|_{\omega_{\mathbf{a}}}+\left\|p-p_{h}^{k}\right\|_{\omega_{\mathbf{a}}}\right)\left\|\nabla\left(\boldsymbol{\psi}_{\mathbf{a}, m} v\right)\right\|_{\omega_{\mathbf{a}}} \\
& +\left\|\left(\mathrm{R}_{\mathbf{a}}^{k, i}\right)_{m}\left|\omega_{\mathbf{a}}\right|^{-1}\right\|_{\omega_{\mathbf{a}}}\|v\|_{\omega_{\mathbf{a}}} \\
\leq & \max \left\{1, \beta^{-1}\right\}\left(\left\|\nabla\left(\mathbf{u}-\mathbf{u}_{h}^{k, i}\right)\right\|_{\omega_{\mathbf{a}}}+\beta\left\|p-p_{h}^{k}\right\|_{\omega_{\mathbf{a}}}\right)\left\|\nabla\left(\boldsymbol{\psi}_{\mathbf{a}, m} v\right)\right\|_{\omega_{\mathbf{a}}} \\
& +\left|\left(\mathrm{R}_{\mathbf{a}}^{k, i}\right)_{m}\right|\left|\omega_{\mathbf{a}}\right|^{-1 / 2}\|v\|_{\omega_{\mathbf{a}}},
\end{aligned}
$$

where we used the fact that $\left(\mathbf{f}, \boldsymbol{\psi}_{\mathbf{a}, m} v\right)_{\omega_{\mathbf{a}}}=\left(\mathbf{f}, \boldsymbol{\psi}_{\mathbf{a}, m} v\right)_{\Omega}$ as $\boldsymbol{\psi}_{\mathbf{a}, m} v=0$ on the boundary of $\omega_{\mathbf{a}}$ and the characterization 1.3a of the weak solution. Moreover, employing $\|\nabla v\|_{\omega_{\mathbf{a}}}=1$ and $\left\|\boldsymbol{\psi}_{\mathbf{a}, m}\right\|_{\omega_{\mathbf{a}}, \infty}=1$, we have

$$
\begin{aligned}
\left\|\nabla\left(\boldsymbol{\psi}_{\mathbf{a}, m} v\right)\right\|_{\omega_{\mathbf{a}}} & =\left\|\nabla \boldsymbol{\psi}_{\mathbf{a}, m} v+\boldsymbol{\psi}_{\mathbf{a}, m} \nabla v\right\|_{\omega_{\mathbf{a}}} \\
& \leq\left\|\nabla \boldsymbol{\psi}_{\mathbf{a}, m}\right\|_{\omega_{\mathbf{a}}, \infty}\|v\|_{\omega_{\mathbf{a}}}+\left\|\boldsymbol{\psi}_{\mathbf{a}, m}\right\|_{\omega_{\mathbf{a}, \infty}}\|\nabla v\|_{\omega_{\mathbf{a}}} \\
& \leq C_{\mathrm{PF}, \omega_{\mathbf{a}}} h_{\omega_{\mathbf{a}}}\left\|\nabla \psi_{\mathbf{a}, m}\right\|_{\omega_{\mathbf{a}}, \infty}+1,
\end{aligned}
$$

where on the patch $\omega_{\mathbf{a}}$ for $\mathbf{a} \in \mathcal{V}_{h}^{\text {int }}$, we use the Poincaré inequality, cf. 6.1), and for $\mathbf{a} \in \mathcal{V}_{h}^{\text {ext }}$ the Friedrichs inequality, cf. 6.2 , to infer

$$
\|v\|_{\omega_{\mathbf{a}}} \leq C_{\mathrm{PF}, \omega_{\mathbf{a}}} h_{\omega_{\mathbf{a}}}\|\nabla v\|_{\omega_{\mathbf{a}}}, \quad \forall v \in H_{*}^{1}\left(\omega_{\mathbf{a}}\right) .
$$

For any $\mathbf{a} \in \mathcal{V}_{h}^{\text {int }}$, relying on 5.3 and $1.3 \mathrm{a}$, we have

$$
\begin{aligned}
\left(\mathrm{R}_{\mathbf{a}}^{k, i}\right)_{m}= & \left(\mathbf{f}, \boldsymbol{\psi}_{\mathbf{a}, m}\right)_{\omega_{\mathbf{a}}}-\left(\nabla \mathbf{u}_{h}^{k, i}, \nabla \boldsymbol{\psi}_{\mathbf{a}, m}\right)_{\omega_{\mathbf{a}}}+\left(\nabla \cdot \boldsymbol{\psi}_{\mathbf{a}, m}, p_{h}^{k}\right)_{\omega_{\mathbf{a}}} \\
= & \left(\nabla \mathbf{u}, \nabla \boldsymbol{\psi}_{\mathbf{a}, m}\right)_{\omega_{\mathbf{a}}}-\left(\nabla \cdot \boldsymbol{\psi}_{\mathbf{a}, m}, p\right)_{\omega_{\mathbf{a}}}-\left(\nabla \mathbf{u}_{h}^{k, i}, \nabla \boldsymbol{\psi}_{\mathbf{a}, m}\right)_{\omega_{\mathbf{a}}} \\
& +\left(\nabla \cdot \boldsymbol{\psi}_{\mathbf{a}, m}, p_{h}^{k}\right)_{\omega_{\mathbf{a}}} \\
\leq & \left(\left\|\nabla\left(\mathbf{u}-\mathbf{u}_{h}^{k, i}\right)\right\|_{\omega_{\mathbf{a}}}+\left\|p-p_{h}^{k}\right\|_{\omega_{\mathbf{a}}}\right)\left\|\nabla \boldsymbol{\psi}_{\mathbf{a}, m}\right\|_{\omega_{\mathbf{a}}} \\
\leq & \max \left\{1, \beta^{-1}\right\}\left(\left\|\nabla\left(\mathbf{u}-\mathbf{u}_{h}^{k, i}\right)\right\|_{\omega_{\mathbf{a}}}+\beta\left\|p-p_{h}^{k}\right\|_{\omega_{\mathbf{a}}}\right)\left\|\nabla \boldsymbol{\psi}_{\mathbf{a}, m}\right\|_{\omega_{\mathbf{a}}, \infty}\left|\omega_{\mathbf{a}}\right|^{1 / 2} .
\end{aligned}
$$

Employing again (7.5) together with 7.3) -7.4 yields 7.2 .

Step 2: Still for $\mathbf{a} \in \mathcal{V}_{K}$, let $\tilde{r}_{\mathbf{a}, m} \in H_{*}\left(\omega_{\mathbf{a}}\right), 1 \leq m \leq d$, be defined by

$$
\begin{aligned}
& \left(\nabla \tilde{r}_{\mathbf{a}, m}, \nabla v\right)_{\omega_{\mathbf{a}}} \\
= & -\left(\left(\nabla \mathbf{u}_{h}^{k, i}-p_{h}^{k} \mathbb{I}\right) \boldsymbol{\psi}_{\mathbf{a}, m}, \nabla v\right)_{\omega_{\mathbf{a}}}+\left(\boldsymbol{\Pi}_{l}\left(\mathbf{f} \cdot \boldsymbol{\psi}_{\mathbf{a}, m}\right)\right. \\
& \left.-\left(\nabla \mathbf{u}_{h}^{k, i}-p_{h}^{k} \mathbb{I}\right): \nabla \boldsymbol{\psi}_{\mathbf{a}, m}-\left(\mathrm{R}_{\mathbf{a}}^{k, i}\right)_{m}\left|\omega_{\mathbf{a}}\right|^{-1}, v\right)_{\omega_{\mathbf{a}}}
\end{aligned}
$$

for all $v \in H_{*}^{1}\left(\omega_{\mathbf{a}}\right)$. Using that this problem, in contrast to (7.1), has piecewise polynomial data and that the equilibration of Definition 5.1 is done with the corresponding degree $l$ and componentwise, we can use [14, Theorem 7] on triangles or rectangular parallelepipeds and [31, Corollaries 3.3 and 3.8] on tetrahedra to infer that, for $1 \leq m \leq d$,

$$
\left\|\left(\left(\nabla \mathbf{u}_{h}^{k, i}-p_{h}^{k} \mathbb{I}\right) \psi_{\mathbf{a}}-\mathbb{d}_{\mathbf{a}}^{k, i}\right)_{m}\right\|_{\omega_{\mathbf{a}}} \leq C_{\mathrm{st}}\left\|\nabla \tilde{r}_{\mathbf{a}, m}\right\|_{\omega_{\mathbf{a}}},
$$


with the constant $C_{\mathrm{st}}$ only depending on the shape regularity parameter $\kappa_{\mathcal{T}}$. Finally, using

$$
\begin{aligned}
& \sup _{v \in H_{*}^{1}\left(\omega_{\mathbf{a}}\right) ;\|\nabla v\|_{\omega_{\mathbf{a}}}=1}\left(\boldsymbol{\Pi}_{l}\left(\mathbf{f} \cdot \boldsymbol{\psi}_{\mathbf{a}, m}\right)-\mathbf{f} \cdot \boldsymbol{\psi}_{\mathbf{a}, m}, v\right)_{\omega_{\mathbf{a}}} \\
= & \sup _{v \in H_{*}^{1}\left(\omega_{\mathbf{a}}\right) ;\|\nabla v\|_{\omega_{\mathbf{a}}}=1}\left\{\sum_{K^{\prime} \in \omega_{\mathbf{a}}}\left(\boldsymbol{\Pi}_{l}\left(\mathbf{f} \cdot \boldsymbol{\psi}_{\mathbf{a}, m}\right)-\mathbf{f} \cdot \boldsymbol{\psi}_{\mathbf{a}, m}, v-v_{K}\right)_{K^{\prime}}\right\} \\
\leq & \left\{\sum_{K^{\prime} \in \omega_{\mathbf{a}}}\left(\frac{h_{K^{\prime}}}{\pi}\left\|\boldsymbol{\Pi}_{l}\left(\mathbf{f} \cdot \boldsymbol{\psi}_{\mathbf{a}, m}\right)-\mathbf{f} \cdot \boldsymbol{\psi}_{\mathbf{a}, m}\right\|_{K^{\prime}}\right)^{2}\right\}^{1 / 2}
\end{aligned}
$$

leads to

$$
\begin{aligned}
& \left\|\left(\left(\nabla \mathbf{u}_{h}^{k, i}-p_{h}^{k} \mathbb{I}\right) \psi_{\mathbf{a}}-\mathbb{d}_{\mathbf{a}}^{k, i}\right)_{m}\right\|_{\omega_{\mathbf{a}}} \\
\leq & C_{\mathrm{st}}\left\|\nabla r_{\mathbf{a}, m}\right\|_{\omega_{\mathbf{a}}}+C_{\mathrm{st}}\left\{\sum_{K^{\prime} \in \omega_{\mathbf{a}}}\left(\frac{h_{K^{\prime}}}{\pi}\left\|\boldsymbol{\Pi}_{l}\left(\mathbf{f} \cdot \boldsymbol{\psi}_{\mathbf{a}, m}\right)-\mathbf{f} \cdot \boldsymbol{\psi}_{\mathbf{a}, m}\right\|_{K^{\prime}}\right)^{2}\right\}^{1 / 2} .
\end{aligned}
$$

Step 3: Employing 5.2 and the fact $\left.\sum_{\mathbf{a} \in \mathcal{V}_{K}} \psi_{\mathbf{a}}\right|_{K}=1$, we get

$$
\begin{aligned}
\left\|\left(\nabla \mathbf{u}_{h}^{k, i}-p_{h}^{k} \mathbb{I}-\mathbb{d}_{h}^{k, i}\right)_{m}\right\|_{K} & =\left\|\sum_{\mathbf{a} \in \mathcal{V}_{K}}\left(\left(\nabla \mathbf{u}_{h}^{k, i}-p_{h}^{k} \mathbb{I}\right) \psi_{\mathbf{a}}-\mathbb{d}_{\mathbf{a}}^{k, i}\right)_{m}\right\|_{K} \\
& \leq \sum_{\mathbf{a} \in \mathcal{V}_{K}}\left\|\left(\left(\nabla \mathbf{u}_{h}^{k, i}-p_{h}^{k} \mathbb{I}\right) \psi_{\mathbf{a}}-\mathbb{d}_{\mathbf{a}}^{k, i}\right)_{m}\right\|_{K} \\
& \leq \sum_{\mathbf{a} \in \mathcal{V}_{K}}\left\|\left(\left(\nabla \mathbf{u}_{h}^{k, i}-p_{h}^{k} \mathbb{I}\right) \psi_{\mathbf{a}}-\mathbb{d}_{\mathbf{a}}^{k, i}\right)_{m}\right\|_{\omega_{\mathbf{a}}} .
\end{aligned}
$$

Combining (7.2), (7.7), and 7.8 yields the assertion.

Remark 7.2 (Global efficiency). Global stopping criteria (4.2) in place of the local ones (4.3) are sufficient for the global efficiency.

Remark 7.3 (Single-loop setting of Algorithm 4.3). Adaptation of Theorem 7.1 to the setting of the singleloop Algorithm 4.3 consists again in disregarding the outer iteration index $k$, while relying on the local stopping criteria $\eta_{\mathrm{rem}, K}^{i} \leq \gamma_{\mathrm{rem}, K}\left(\eta_{\mathrm{alg}, \mathbf{u}, K}^{i}+\eta_{\mathrm{alg}, p, K}^{i}\right)$ and $\left(\eta_{\mathrm{alg}, \mathbf{u}, K}^{i}+\eta_{\mathrm{alg}, p, K}^{i}\right) \leq \gamma_{\mathrm{alg}, K} \eta_{\mathrm{disc}, K}^{i}$. Global effciency then follows under the global stopping criteria 4.5).

\section{Numerical examples}

This section presents a numerical assessment of our a posteriori error estimates and of the proposed adaptive algorithms. We test the Uzawa and the MinRes algebraic solvers on two examples with known analytic solution, a smooth one and another one with a singularity in a reentrant corner of the domain. The discretization is given by the Taylor-Hood conforming finite elements 3.3 of order $l=2$ on triangles. There is, in particular, no stabilization; consequently, the block matrix $\mathbb{A}$ in 1.7 is symmetric, $\mathbb{C}=\mathbb{B}^{t}$, and $\mathbb{D}=0$.

Firstly, we consider three different types of Uzawa approaches with (preconditioned) conjugate gradients (CG) as the inner solver: 1) the "exact" Uzawa method of Algorithm 1.3, where the CG for the system (1.8) is run until the relative algebraic residual gets below $10^{-10}$, and with the outer stopping criterion in step (1.12) being $\left.\left\|\mathbb{B U}^{k, i}\right\| \leq 10^{-9} ; 2\right)$ the inexact Uzawa method of [27, i.e., Algorithm 1.3 where the inner stopping criterion in step (1.10) is $\left\|\mathrm{R}^{k, i}\right\| \leq\left\|\mathbb{B U}^{k, i}\right\|$ and the outer stopping criterion is as above; 3 ) our adaptive inexact Uzawa method of Algorithm 4.1 with the global adaptive stopping criteria 4.2 with parameters $\gamma_{\mathrm{rem}}:=1, \gamma_{\mathrm{alg}, \mathbf{u}}:=0.5, \gamma_{\mathrm{alg}, p}:=0.5$ and the choice of the number of additional steps $\nu_{0}:=5$. We set the Uzawa parameter $\alpha:=1$.

Recall that $(\mathbf{u}, p)$ is the exact solution of 1.3$),\left(\mathbf{u}_{h}, p_{h}\right)$ is the solution obtained by the finite element discretization with exact solve of the linear system (1.7), $\left(\mathbf{u}_{h}^{k, i}, p_{h}^{k}\right)$ is the solution obtained on the $k$-th 
Uzawa iteration and the $i$-th linear algebraic iteration on step 2(c)iil), and $\left(\mathbf{u}_{h}^{k, \infty}, p_{h}^{k}\right)$ is obtained via 1.8 on the $k$-th Uzawa iteration with an exact algebraic solve. The different errors components are given as:

$$
\begin{aligned}
& \text { total error }:=\left\|\nabla\left(\mathbf{u}-\mathbf{u}_{h}^{k, i}\right)\right\|+\beta\left\|p-p_{h}^{k}\right\|, \\
& \text { discretization error }:=\left\|\nabla\left(\mathbf{u}-\mathbf{u}_{h}\right)\right\|+\beta\left\|p-p_{h}\right\|, \\
& \text { Uzawa (outer) error }:=\left\|\nabla\left(\mathbf{u}_{h}-\mathbf{u}_{h}^{k, \infty}\right)\right\|+\beta\left\|p_{h}-p_{h}^{k}\right\|, \\
& \text { algebraic (inner) error }:=\left\|\nabla\left(\mathbf{u}_{h}^{k, \infty}-\mathbf{u}_{h}^{k, i}\right)\right\| .
\end{aligned}
$$

Secondly, for the MinRes algorithm, we compare 1) the "exact" MinRes method of Algorithm 1.4 where the relative norm of the total residual vector composed of $\mathrm{R}_{\mathbf{u}}^{i}$ and $\mathrm{R}_{p}^{i}$ is requested to lie below $10^{-9} ; 2$ ) our adaptive MinRes method of Algorithm 4.3 with the global adaptive stopping criteria (4.5) with $\gamma_{\text {rem }}:=1$, $\gamma_{\text {alg }}:=0.5$, and $\nu_{0}:=5$. In this setting, $\left(\mathbf{u}_{h}^{i}, p_{h}^{i}\right)$ is the solution obtained on the $i$-th step of MinRes iteration. Here we only have three different errors components,

$$
\begin{aligned}
& \text { total error }:=\left\|\nabla\left(\mathbf{u}-\mathbf{u}_{h}^{i}\right)\right\|+\beta\left\|p-p_{h}^{i}\right\|, \\
& \text { discretization error }:=\left\|\nabla\left(\mathbf{u}-\mathbf{u}_{h}\right)\right\|+\beta\left\|p-p_{h}\right\|, \\
& \text { algebraic (inner) error }:=\left\|\nabla\left(\mathbf{u}_{h}-\mathbf{u}_{h}^{i}\right)\right\|+\beta\left\|p_{h}-p_{h}^{i}\right\| .
\end{aligned}
$$

In the following, we employ the cheaper reconstruction of Remark 5.3 of order 1 . The entire implementation has been done with the FreeFem++ scientific calculation code [35.

\subsection{Smooth solution}

We consider here the computational domain as $\Omega=(0,1)^{2}$, with homogeneous Dirichlet boundary conditions. The exact solution is given by, cf. [34, equation 8.1 with parameter $\alpha=1$ ],

$$
\mathbf{u}=\left(\begin{array}{c}
2 x^{2} y(x-1)^{2}(y-1)^{2}+x^{2} y^{2}(2 y-2)(x-1)^{2} \\
-2 x y^{2}(x-1)^{2}(y-1)^{2}-x^{2} y^{2}(2 x-2)(y-1)^{2}
\end{array}\right)
$$

and

$$
p=x+y-1 .
$$

The volumetric force $\mathbf{f}$ is chosen accordingly. We consider eight levels of uniform mesh refinement. The inf-sup constant $\beta$ is approximately equal to 0.44 here, see [34] for a discussion.

\subsubsection{Uzawa algorithms}

We first consider no preconditioning of the CG inner solver. Figure1 1 presents the total error and the various estimators as a function of the number of mesh elements. It can be seen that the three approaches yield almost indistinguishable value for the total error; in this smooth case, the convergence order is $O\left(h^{2}\right)$. For the exact and inexact Uzawa method, the "Uzawa" estimators $\eta_{\mathrm{alg}, p}^{k, i}$ take the values around $10^{-7}$, whereas the "algebraic" estimators $\eta_{\text {alg, }}^{k, i}$ are much smaller in the exact case than in the inexact one. For the adaptive inexact method of Algorithm 4.1. we terminate the computation with much larger $\eta_{\text {alg, }, p}^{k, i}$ and $\eta_{\text {alg,u }}^{k, i}$ estimators, just sufficient not to influence the total error. For all the three methods, the total estimators converge with the same speed as the total error, as proven in Theorem 7.1 (cf. also Remark 7.2).

Figure 2 focuses on the finest mesh, and displays the dependence of the total error and of the various estimators on the Uzawa iteration. The total error and the total estimator decrease rapidly for the first 30-40 Uzawa iterations and then stagnate, since therefrom the influence of the Uzawa iteration error gets negligible. This is precisely the point where our adaptive inexact Uzawa method stops, leading to an important economy of the Uzawa iterations necessary.

In Figure 3 we compare the overall performance of the three approaches. We present the number of Uzawa iterations as a function of the refinement level, the number of algebraic solver (CG) iterations as a function of Uzawa iterations on the last refined mesh, and the total number of algebraic solver iterations as a function of the refinement level. Concerning the number of Uzawa iterations, the inexact method needs 


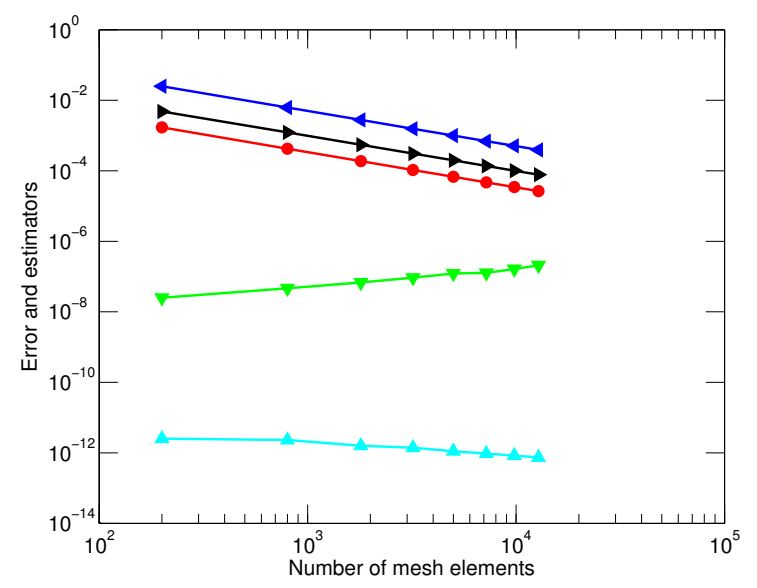

(a) Exact

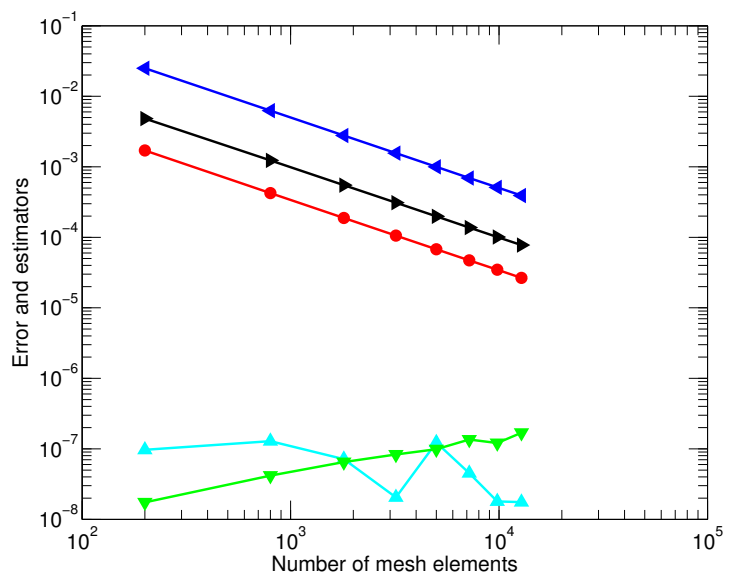

(b) Inexact

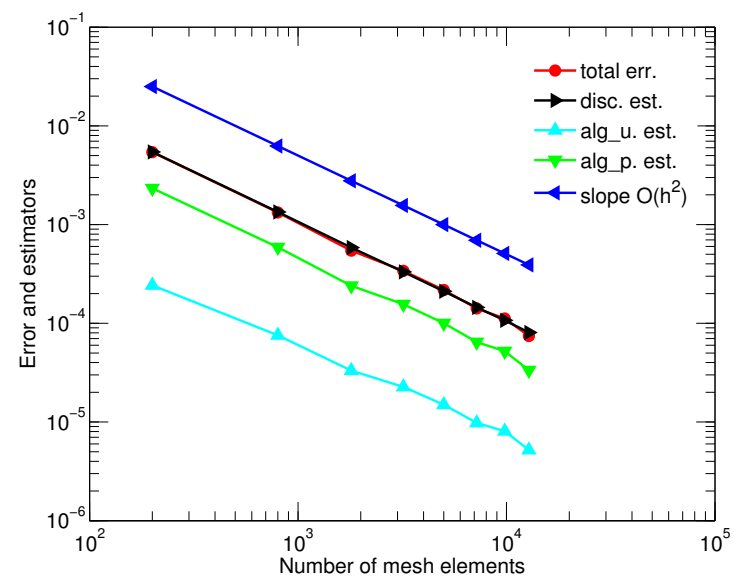

(c) Adaptive inexact

Figure 1: Error and estimators on uniformly refined meshes, unpreconditioned CG-Uzawa solver

sometimes more iterations than the exact one, with, though, only few inner algebraic iterations for the last 30-40 Uzawa iterations, while the adaptive inexact method reduces this number by a significant factor. On each outer Uzawa iteration step, the adaptive inexact method also needs fewer CG iterations than the two other approaches. In combination, the adaptive inexact method needs the smallest number of total algebraic solver iterations; on the eighth refined mesh, the three approaches need respectively 24443, 11374, and 6360 total algebraic solver iterations.

The total effectivity indices for the three approaches are presented in Figure 4 There are computed as the ratio between the total estimator and the total error, i.e.,

$$
\text { total effectivity index }:=\frac{2\left(\eta_{\mathrm{F}}^{k, i}+\eta_{\mathrm{D}}^{k, i}+\eta_{\mathrm{rem}}^{k, i}+\eta_{\mathrm{osc}}^{k, i}\right)}{\text { total error }},
$$

see (6.4). This index for the adaptive inexact Uzawa algorithm appears to be lower than for the two other considered algorithms; setting, however, the parameters $\gamma_{\mathrm{rem}}, \gamma_{\mathrm{alg}, p}$, and $\gamma_{\mathrm{alg}, \mathbf{u}}$ smaller, we obtain almost the same effectivity indices.

Similarly as for the total effectivity index, we also define the effectivity indices for each error component in the adaptive inexact method:

$$
\text { discretization effectivity index }:=\frac{\eta_{\text {disc }}^{k, i}}{\text { discretization error }},
$$




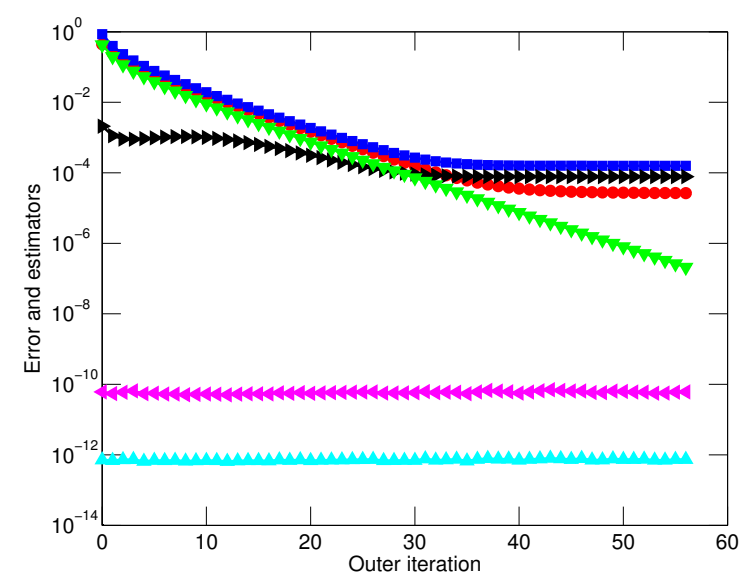

(a) Exact

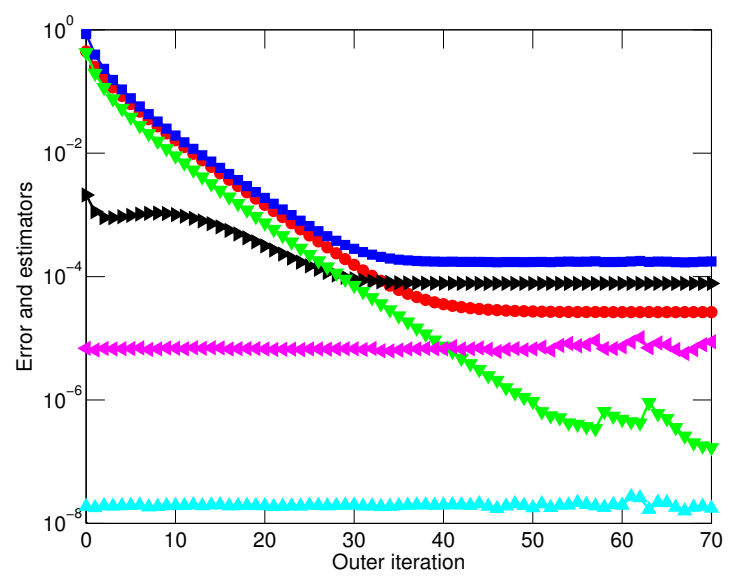

(b) Inexact

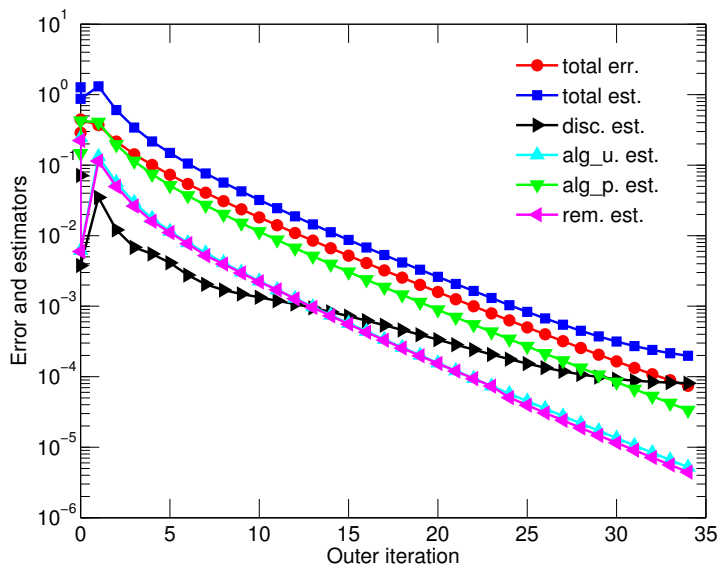

(c) Adaptive inexact

Figure 2: Error and estimators as a function of Uzawa iterations

$$
\begin{aligned}
\text { Uzawa effectivity index } & :=\frac{\eta_{\text {alg, }, p}^{k, i}}{\text { Uzawa error }}, \\
\text { algebraic effectivity index } & :=\frac{\eta_{\text {alg,u }}^{k, i}}{\text { algebraic error }} .
\end{aligned}
$$

We can see in Figure 4, right, that all of them take values nicely close to the optimal value of one; note that the total estimate can indeed be smaller than the discretization estimate, compare 6.4 with 6.13).

Finally, in Figure 5, we display the spatial distribution of the different error components (left) and of the corresponding estimators (right), on the eighth refined mesh. We can observe a nice match.

We now consider the use of the classical incomplete Cholesky factorization preconditioner for the CG solver with drop-off tolerance equal to $10^{-4}$. The results for our adaptive inexact algorithm can be found in Figure 6. We can conclude that the preconditioner has a significant influence on the number of CG iterations at each Uzawa step, but not on the number of Uzawa iterations. Our adaptive inexact algorithm reduces here the total number of iterations by a factor of 10 in comparison with the exact one. The effectivity indices take here similar values as in Figure 4 and the estimates again match the error componentwise as in Figure 5 (not shown). 


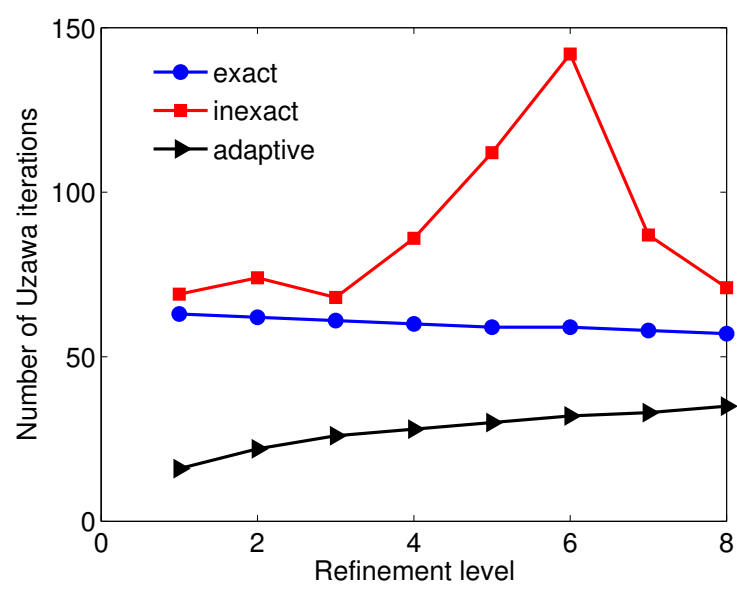

(a) Uzawa iterations per refinement level

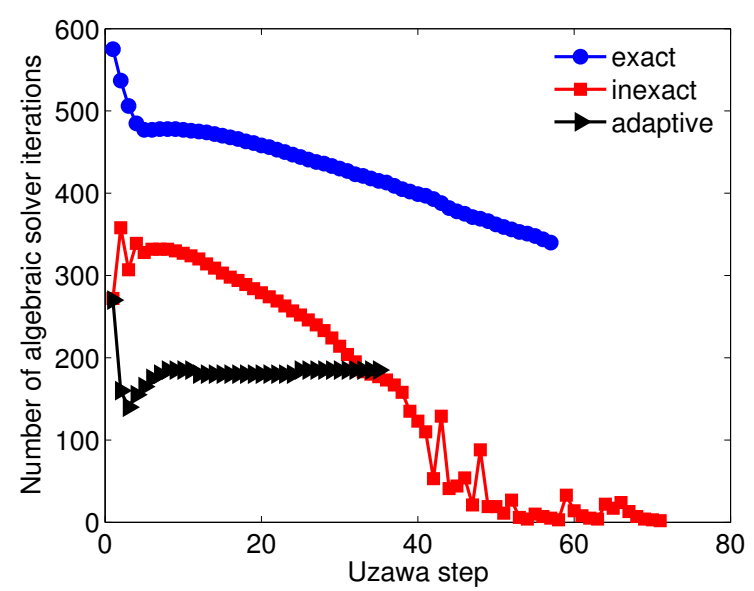

(b) CG iterations per Uzawa step, 8th mesh

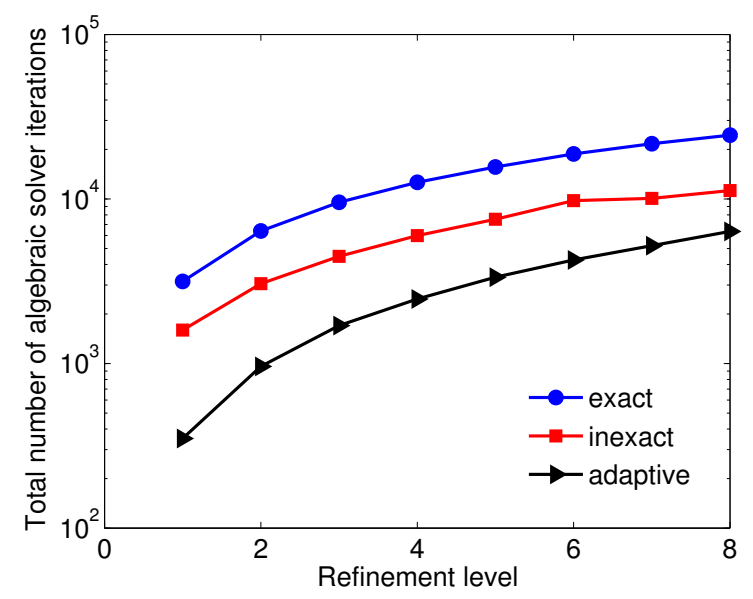

(c) CG iterations per refinement level

Figure 3: Number of unpreconditioned CG and Uzawa iterations

\subsubsection{MinRes algorithms}

With the same analytic example, the performance of our adaptive MinRes Algorithm 4.3 is showcased in the following; we henceforth only consider the preconditionined case. As the the system matrix (1.7) is symmetric but not positive definite, we use block preconditioning of the form [28],

$$
M=\left(\begin{array}{ll}
\mathbb{P} & 0 \\
0 & \mathbb{I}
\end{array}\right)
$$

for the choice of $\mathbb{P} \in \mathbb{R}^{M \times M}$, we use the incomplete Cholesky factorization with drop-off tolerance equal to $10^{-4}$ similarly to the the Uzawa case. The definition of the total effectivity indices is the same as in the Uzawa case; for each error component, we use here

$$
\begin{aligned}
\text { discretization effectivity index } & :=\frac{\eta_{\text {disc }}^{i}}{\text { discretization er }} \\
\text { algebraic effectivity index } & :=\frac{\eta_{\text {alg }, \mathbf{u}}^{i}+\eta_{\text {alg }, p}^{i}}{\text { algebraic error }} .
\end{aligned}
$$




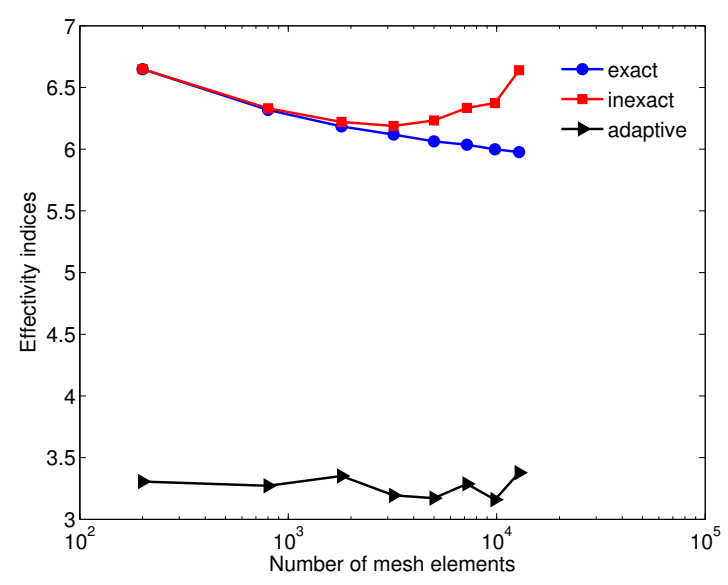

(a) Exact, inexact, and adaptive inexact case

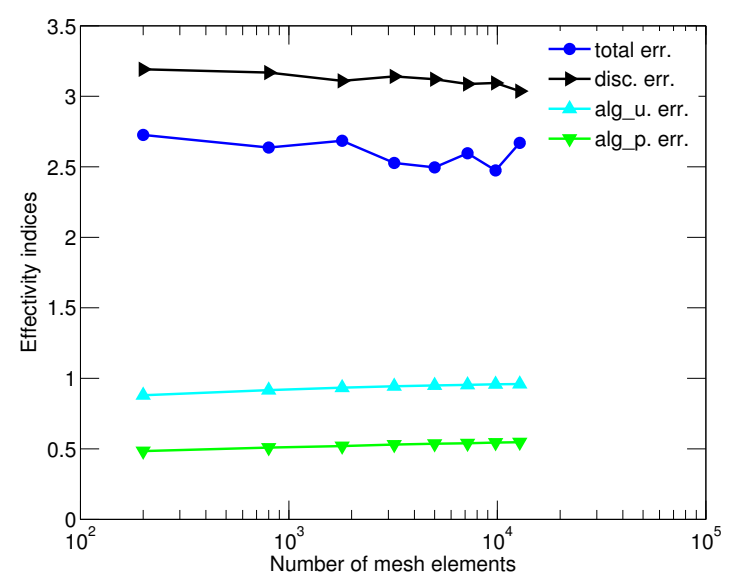

(b) Error components, adaptive inexact case

Figure 4: Effectivity indices for the unpreconditioned CG-Uzawa algorithms

Overall performance of our adaptive MinRes algorithm is shown in Figure 7. Similar conclusions as above can be drawn, namely in terms of identification of the different error components. Somewhat higher effectivity indices are observed, though. For higher-level mesh refinement, our criterion $4.5 \mathrm{~b}$ for stopping the MinRes solver seems to correlate with requesting the relative algebraic residual being below $10^{-9}$. For this reason, virtually no economy in terms of the number of iterations can be seen in Subfigure 7(c). Recall, though, that our criteria choose automatically an adequate stopping point in any situation, whereas $10^{-9}$ for the relative algebraic residual is just an ad hoc choice. In Figure 8, we display the spatial distribution of the two error components (left) and of the corresponding estimators (right), on the eighth refined mesh. A nice match can again be observed.

\subsection{Singular solution}

We consider here the $L$-shaped domain $\Omega=(-1,1)^{2} \backslash[0,1] \times[-1,0]$, with an inhomogeneous Dirichlet boundary condition prescribed by the analytic solution, given in given in polar coordinates by, cf. 9,

$$
\mathbf{u}(r, \varphi)=r^{\kappa}\left[\begin{array}{l}
\cos (\varphi) \psi^{\prime}(\varphi)+(1+\kappa) \sin (\varphi) \psi(\varphi) \\
\sin (\varphi) \psi^{\prime}(\varphi)-(1+\kappa) \cos (\varphi) \psi(\varphi)
\end{array}\right]
$$

and

$$
p(r, \varphi)=-r^{\kappa-1} \frac{(1+\kappa)^{2} \psi^{\prime}(\varphi)+\psi^{\prime \prime \prime}(\varphi)}{1-\kappa}
$$

with the function $\psi(\varphi)$ defined as

$$
\begin{aligned}
\psi(\varphi)= & \frac{\sin ((1+\kappa) \varphi) \cos (\kappa \omega)}{1+\kappa}-\cos ((1+\kappa) \varphi)+\frac{\sin ((\kappa-1) \varphi) \cos (\kappa \omega)}{1-\kappa} \\
& -\cos ((\kappa-1) \varphi) .
\end{aligned}
$$

We consider $\kappa=856399 / 1572864 \approx 0.54$ and $\omega=3 \pi / 2$. There is the typical corner singularity in the re-entrant corner $(0,0)$. The volumetric force is here $\mathbf{f}=\mathbf{0}$ and the inf-sup constant $\beta$ is used with value 0.3 , see 25 and the references therein. Similarly to the preceding example, we consider eight levels of uniform mesh refinement.

\subsubsection{Uzawa algorithms}

The overall performance of the adaptive inexact preconditioned CG-Uzawa algorithm in this test can be appreciated in Figure 9. The estimators and total error as a function of the number of mesh elements are in 
particular shown in Figure 9(a). The two other approaches (exact and inexact) yield almost indistinguishable values for the total error and estimator for this test case as well, while the algebraic and Uzawa estimators $\eta_{\mathrm{alg}, p}^{k, i}$ and $\eta_{\mathrm{alg}, \mathbf{u}}^{k, i}$ differ significantly between them, similarly to Figure 1 (not presented). The convergence order here is $O\left(h^{\kappa}\right)$. Detailed behavior of the different estimators is summarized in Figure 9(b). Very decent effectivity indices can be found in Figure 9(c); in particular, the singularity has no influence on the quality of our estimators even in presence of inexact solvers (with adaptive stopping criteria (4.2), in complete agreement with the theory. The rather important savings in the number of CG and Uzawa iterations can be appreciated in Figure 10. In particular, our adaptive inexact Uzawa method needs only around 5\% iterations of the exact one. On the eighth refined mesh, the three approaches need respectively 8321, 6518, and 405 total algebraic solver iterations. Finally, the spatial distribution of the developed estimates again matches the different error components very well (except for the Uzawa error, where the main ambiguity lies in its definition itself), see Figure 11

\subsubsection{MinRes algorithms}

We finally test the preconditioned MinRes algorithm. An overall assessment can be found in Figure 12 while Figure 13 presents the actual and estimated error components distributions. It appears in particular that rather important computational savings in terms of the number of iterations are achieved here, in contrast to the case of regular exact solution.

\section{References}

[1] Ainsworth, M., Allendes, A., Barrenechea, G. R., And Rankin, R. On the adaptive selection of the parameter in stabilized finite element approximations. SIAM J. Numer. Anal. 51, 3 (2013), 1585-1609.

[2] Ainsworth, M., And Oden, J. T. A posteriori error estimators for the Stokes and Oseen equations. SIAM J. Numer. Anal. 34, 1 (1997), 228-245.

[3] Anjam, I., Nokka, M., And RePin, S. I. On a posteriori error bounds for approximations of the generalized Stokes problem generated by the Uzawa algorithm. Russian J. Numer. Anal. Math. Modelling 27, 4 (2012), 321-338.

[4] Arioli, M., Liesen, J., Międlar, A., And Strakoš, Z. Interplay between discretization and algebraic computation in adaptive numerical solution of elliptic PDE problems. GAMM-Mitt. 36, 1 (2013), 102-129.

[5] Arnold, D. N., Brezzi, F., And Fortin, M. A stable finite element for the Stokes equations. Calcolo 21, 4 (1984), 337-344 (1985).

[6] Arrow, K. J., Hurwicz, L., and Uzawa, H. Studies in linear and non-linear programming. With contributions by H. B. Chenery, S. M. Johnson, S. Karlin, T. Marschak, R. M. Solow. Stanford Mathematical Studies in the Social Sciences, vol. II. Stanford University Press, Stanford, Calif., 1958.

[7] Bacuta, C. A unified approach for Uzawa algorithms. SIAM J. Numer. Anal. 44, 6 (2006), 2633-2649.

[8] Bank, R. E., and Welfert, B. D. A posteriori error estimates for the Stokes problem. SIAM J. Numer. Anal. 28, 3 (1991), 591-623.

[9] Bänsch, E., Morin, P., And Nochetto, R. H. An adaptive Uzawa FEM for the Stokes problem: convergence without the inf-sup condition. SIAM J. Numer. Anal. 40, 4 (2002), 1207-1229.

[10] Becker, R. An adaptive finite element method for the Stokes equations including control of the iteration error. In ENUMATH 97 (Heidelberg). World Sci. Publ., River Edge, NJ, 1998, pp. 609-620.

[11] Becker, R., Johnson, C., And Rannacher, R. Adaptive error control for multigrid finite element methods. Computing 55, 4 (1995), 271-288. 
[12] Bercovier, M., and Pironneau, O. Error estimates for finite element method solution of the Stokes problem in the primitive variables. Numer. Math. 33, 2 (1979), 211-224.

[13] Boffi, D., Brezzi, F., And Fortin, M. Mixed finite element methods and applications, vol. 44 of Springer Series in Computational Mathematics. Springer, Heidelberg, 2013.

[14] Braess, D., Pillwein, V., And SchöBerl, J. Equilibrated residual error estimates are $p$-robust. Comput. Methods Appl. Mech. Engrg. 198, 13-14 (2009), 1189-1197.

[15] Bramble, J. H., Pasciak, J. E., And Vassilev, A. T. Analysis of the inexact Uzawa algorithm for saddle point problems. SIAM J. Numer. Anal. 34, 3 (1997), 1072-1092.

[16] Brezzi, F., And Douglas, JR., J. Stabilized mixed methods for the Stokes problem. Numer. Math. 53, 1-2 (1988), 225-235.

[17] Brezzi, F., ANd Falk, R. S. Stability of higher-order Hood-Taylor methods. SIAM J. Numer. Anal. $\underline{28}, 3$ (1991), 581-590.

[18] Brezzi, F., And Pitkäranta, J. On the stabilization of finite element approximations of the Stokes equations. In Efficient solutions of elliptic systems (Kiel, 1984), vol. 10 of Notes Numer. Fluid Mech. Friedr. Vieweg, Braunschweig, 1984, pp. 11-19.

[19] Carstensen, C., And Funken, S. A. A posteriori error control in low-order finite element discretisations of incompressible stationary flow problems. Math. Comp. 70, 236 (2001), 1353-1381.

[20] Cheng, X.-L., And Zou, J. An inexact Uzawa-type iterative method for solving saddle point problems. Int. J. Comput. Math. 80, 1 (2003), 55-64.

[21] Destuynder, P., and Métivet, B. Explicit error bounds in a conforming finite element method. Math. Comp. 68, 228 (1999), 1379-1396.

[22] Dobrowolski, M. On the LBB condition in the numerical analysis of the Stokes equations. Applied numerical mathematics 54, 3-4 (2005), 314-323.

[23] Dohrmann, C. R., And Bochev, P. B. A stabilized finite element method for the Stokes problem based on polynomial pressure projections. Internat. J. Numer. Methods Fluids 46, 2 (2004), 183-201.

[24] Dolejší, V., Ern, A., And Vohralík, M. hp-adaptation driven by polynomial-degree-robust a posteriori error estimates for elliptic problems. SIAM J. Sci. Comput. 38, 5 (2016), A3220-A3246.

[25] Dörfler, W., And Ainsworth, M. Reliable a posteriori error control for nonconformal finite element approximation of Stokes flow. Math. Comp. 74, 252 (2005), 1599-1619.

[26] Douglas, JR., J., And Wang, J. P. An absolutely stabilized finite element method for the Stokes problem. Math. Comp. 52, 186 (1989), 495-508.

[27] Elman, H. C., And Golub, G. H. Inexact and preconditioned Uzawa algorithms for saddle point problems. SIAM J. Numer. Anal. 31, 6 (1994), 1645-1661.

[28] Elman, H. C., Silvester, D. J., And Wathen, A. J. Finite elements and fast iterative solvers: with applications in incompressible fluid dynamics, second ed. Numerical Mathematics and Scientific Computation. Oxford University Press, Oxford, 2014.

[29] ERn, A., AND Vohralík, M. Adaptive inexact Newton methods with a posteriori stopping criteria for nonlinear diffusion PDEs. SIAM J. Sci. Comput. 35, 4 (2013), A1761-A1791.

[30] Ern, A., And Vohralík, M. Polynomial-degree-robust a posteriori estimates in a unified setting for conforming, nonconforming, discontinuous Galerkin, and mixed discretizations. SIAM J. Numer. Anal. 53, 2 (2015), 1058-1081. 
[31] Ern, A., And Vohralík, M. Stable broken $H^{1}$ and $\boldsymbol{H}$ (div) polynomial extensions for polynomialdegree-robust potential and flux reconstruction in three space dimensions. HAL Preprint 01422204, submitted for publication, 2016.

[32] Franca, L. P., And Stenberg, R. Error analysis of Galerkin least squares methods for the elasticity equations. SIAM J. Numer. Anal. 28, 6 (1991), 1680-1697.

[33] Girault, V., and Raviart, P.-A. Finite element methods for Navier-Stokes equations, vol. 5 of Springer Series in Computational Mathematics. Springer-Verlag, Berlin, 1986. Theory and algorithms.

[34] Hannukainen, A., Stenberg, R., and Vohralík, M. A unified framework for a posteriori error estimation for the Stokes problem. Numer. Math. 122, 4 (2012), 725-769.

[35] Hеснт, F. New development in FreeFem++. J. Numer. Math. 20, 3-4 (2012), 251-265.

[36] Hughes, T. J. R., And FrancA, L. P. A new finite element formulation for computational fluid dynamics. VII. The Stokes problem with various well-posed boundary conditions: symmetric formulations that converge for all velocity/pressure spaces. Comput. Methods Appl. Mech. Engrg. 65, 1 (1987), $85-96$.

[37] Hughes, T. J. R., Franca, L. P., and Balestra, M. A new finite element formulation for computational fluid dynamics. V. Circumventing the Babuška-Brezzi condition: a stable Petrov-Galerkin formulation of the Stokes problem accommodating equal-order interpolations. Comput. Methods Appl. Mech. Engrg. 59, 1 (1986), 85-99.

[38] Jiránek, P., Strakoš, Z., And Vohralík, M. A posteriori error estimates including algebraic error and stopping criteria for iterative solvers. SIAM J. Sci. Comput. 32, 3 (2010), 1567-1590.

[39] Kay, D., And Silvester, D. A posteriori error estimation for stabilized mixed approximations of the Stokes equations. SIAM J. Sci. Comput. 21, 4 (1999/00), 1321-1336.

[40] Kechkar, N., and Silvester, D. Analysis of locally stabilized mixed finite element methods for the Stokes problem. Math. Comp. 58, 197 (1992), 1-10.

[41] KIM, K.-Y., AND LEE, H.-C. A posteriori error estimators for nonconforming finite element methods of the linear elasticity problem. Journal of computational and applied mathematics 235, 1 (2010), $186-202$.

[42] Lin, Y. On an inexact Uzawa-type algorithm for stabilized saddle point problems. Int. J. Comput. Math. 87, 13 (2010), 2945-2952.

[43] Lin, Y., AND WEI, Y. Corrected Uzawa methods for solving large nonsymmetric saddle point problems. Appl. Math. Comput. 183, 2 (2006), 1108-1120.

[44] Meidner, D., Rannacher, R., and Vihharev, J. Goal-oriented error control of the iterative solution of finite element equations. J. Numer. Math. 17, 2 (2009), 143-172.

[45] Paige, C. C., And Saunders, M. A. Solutions of sparse indefinite systems of linear equations. SIAM J. Numer. Anal. 12, 4 (1975), 617-629.

[46] Payne, L. E., and Weinberger, H. F. An optimal Poincaré inequality for convex domains. Arch. Rational Mech. Anal. 5 (1960), 286-292 (1960).

[47] Pironneau, O. Finite element methods for fluids. John Wiley \& Sons Ltd., Chichester, 1989. Translated from the French.

[48] Quarteroni, A., And Valli, A. Numerical approximation of partial differential equations, vol. 23 of Springer Series in Computational Mathematics. Springer-Verlag, Berlin, 1994.

[49] Rannacher, R., Westenberger, A., And Wollner, W. Adaptive finite element solution of eigenvalue problems: balancing of discretization and iteration error. J. Numer. Math. 18, 4 (2010), 303-327. 
[50] Repin, S. I. Local a posteriori estimates for the Stokes problem. Zap. Nauchn. Sem. S.-Peterburg. Otdel. Mat. Inst. Steklov. (POMI) 318, Kraev. Zadachi Mat. Fiz. i Smezh. Vopr. Teor. Funkts. 36 [35] (2004), 233-245, 312-313.

[51] Silvester, D. J., And Simoncini, V. An optimal iterative solver for symmetric indefinite systems stemming from mixed approximation. ACM Trans. Math. Software 37, 4 (2011), Art. 42, 22.

[52] Song, L., Hou, Y., And CaI, Z. Recovery-based error estimator for stabilized finite element methods for the Stokes equation. Comput. Methods Appl. Mech. Engrg. 272 (2014), 1-16.

[53] Stoyan, G. Towards discrete Velte decompositions and narrow bounds for inf-sup constants. Computers \& Mathematics with Applications 38, 7-8 (1999), 243-261.

[54] TaYlor, C., AND Hood, P. A numerical solution of the Navier-Stokes equations using the finite element technique. Internat. J. Comput. \& Fluids 1, 1 (1973), 73-100.

[55] Verfürth, R. A posteriori error estimators for the Stokes equations. Numer. Math. 55, 3 (1989), 309-325.

[56] Vincent, C., And Boyer, R. A preconditioned conjugate gradient Uzawa-type method for the solution of the Stokes problem by mixed Q1-P0 stabilized finite elements. Internat. J. Numer. Methods Fluids 14, 3 (1992), 289-298.

[57] WANG, J., WANG, Y., AND Ye, X. Unified a posteriori error estimator for finite element methods for the Stokes equations. Int. J. Numer. Anal. Model. 10, 3 (2013), 551-570. 


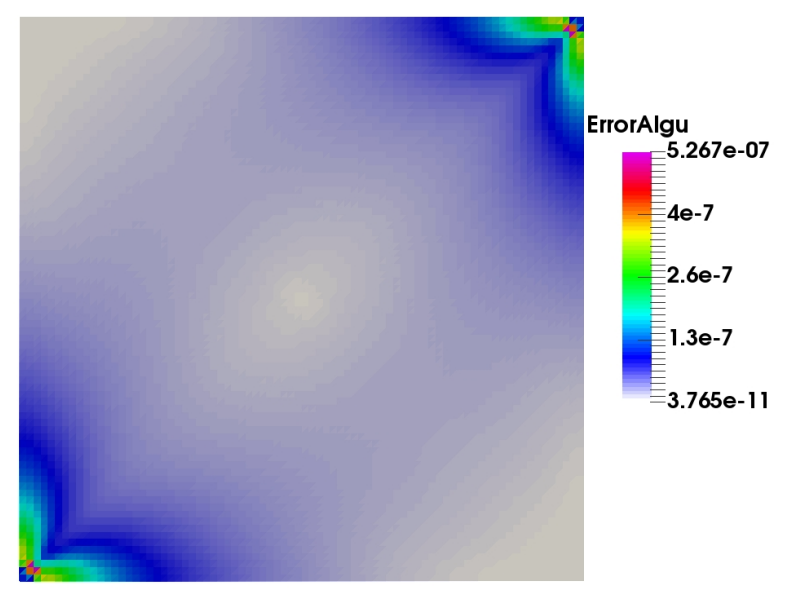

(a) Algebraic error

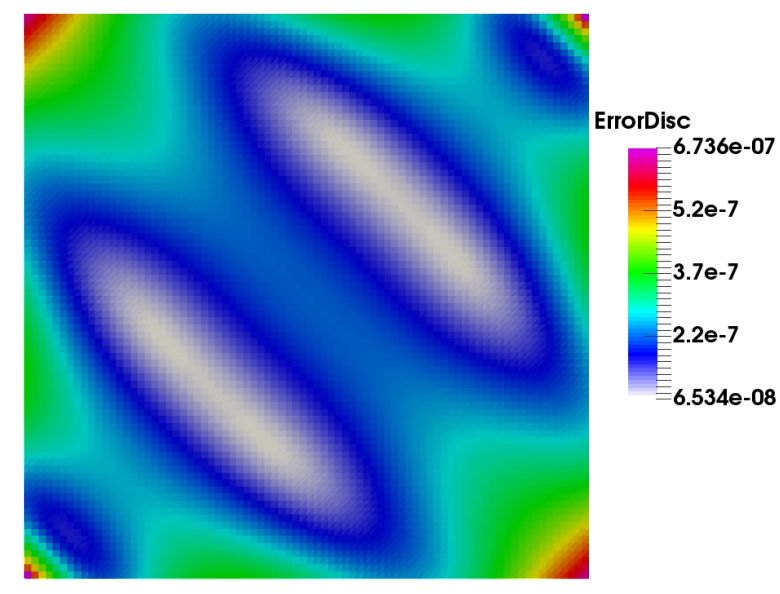

(c) Discretization error

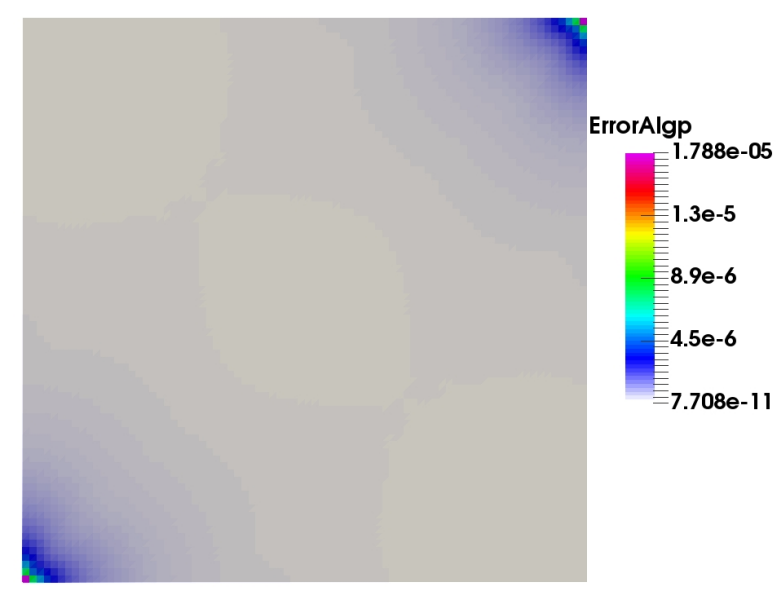

(e) Uzawa error

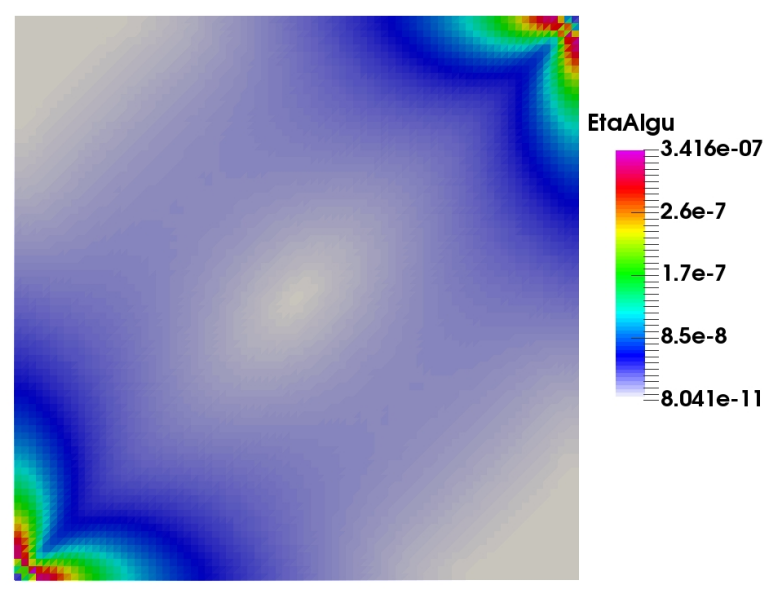

(b) Algebraic estimator

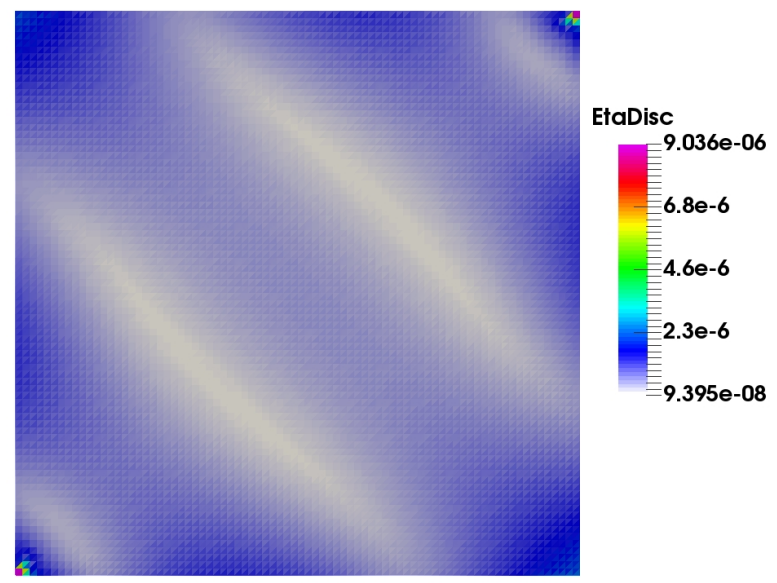

(d) Discretization estimator

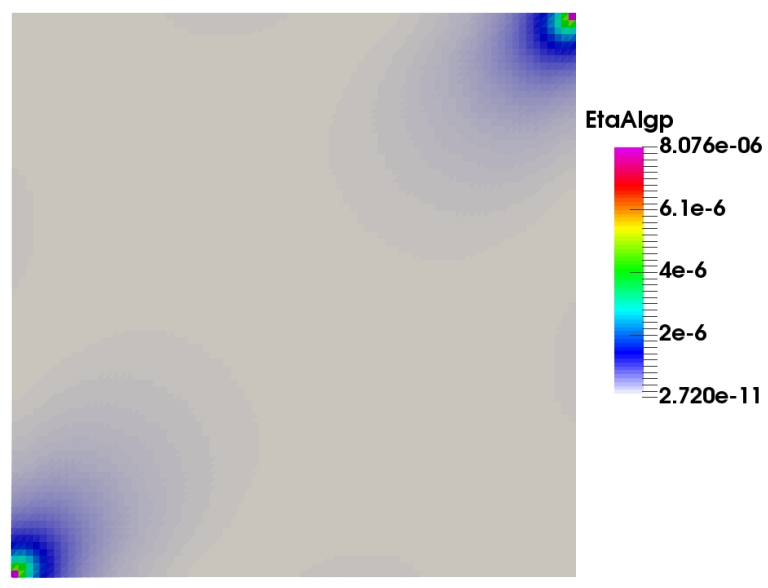

(f) Uzawa estimator

Figure 5: Adaptive inexact unpreconditioned CG-Uzawa algorithm. Spatial distributions of the different error components and corresponding estimates, 8 th 26 mesh 


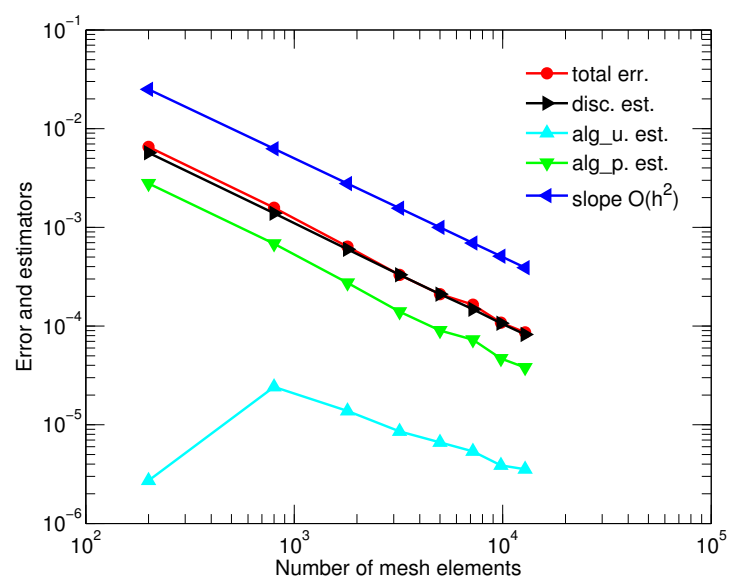

(a) Adaptive inexact Uzawa, un. refinement

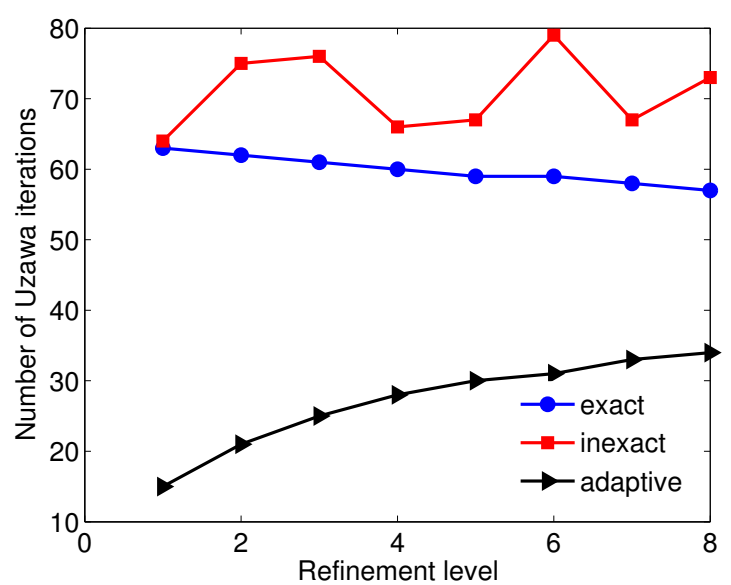

(c) Uzawa iterations per refinement level

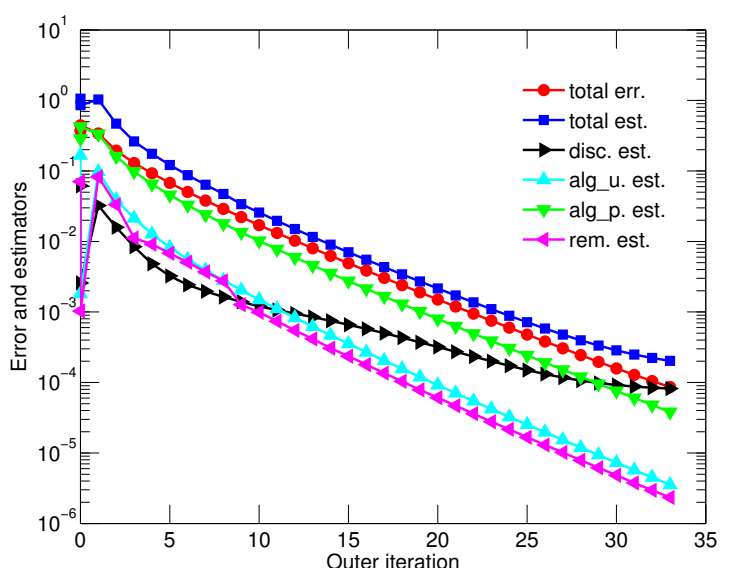

(b) Adaptive inexact Uzawa, 8th mesh

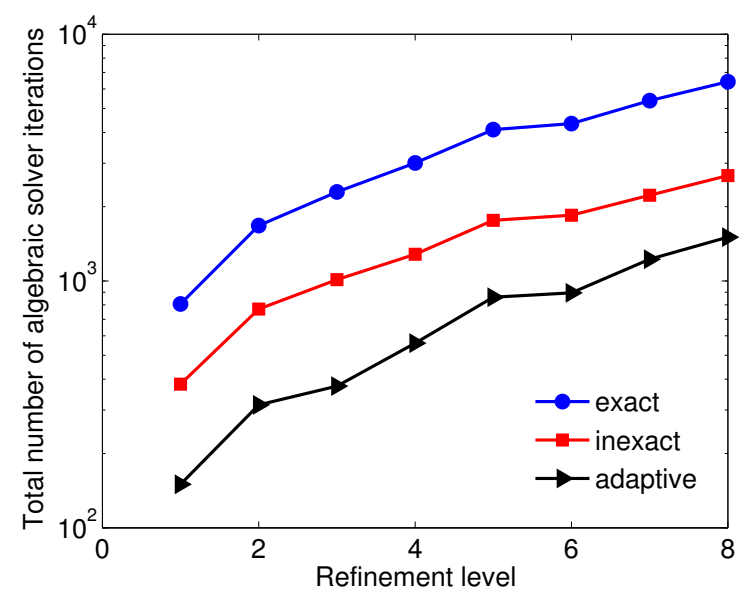

(d) CG iterations per refinement level

Figure 6: Uzawa algorithm, CG with incomplete Cholesky factorization preconditioner 


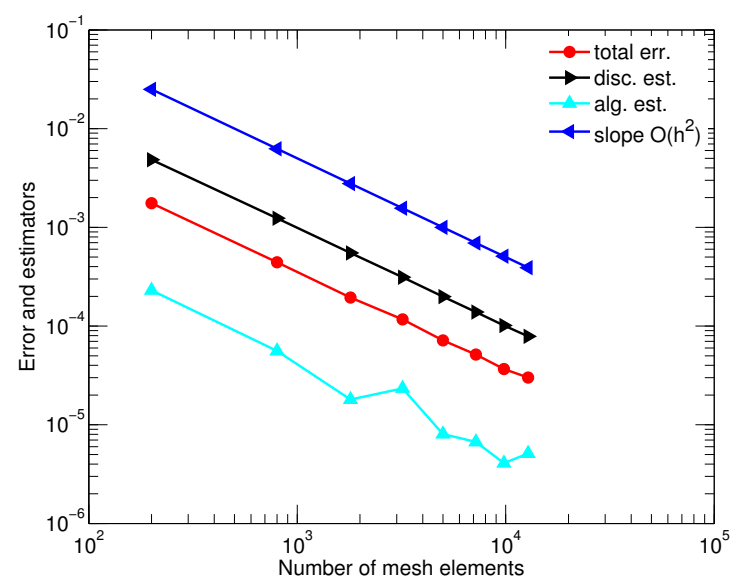

(a) Adaptive inexact MinRes, un. refinement

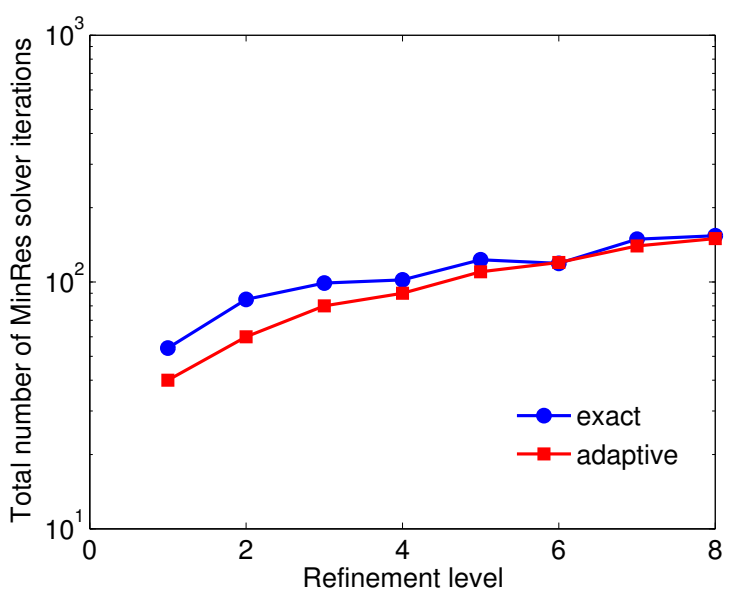

(c) MinRes iterations per refinement level

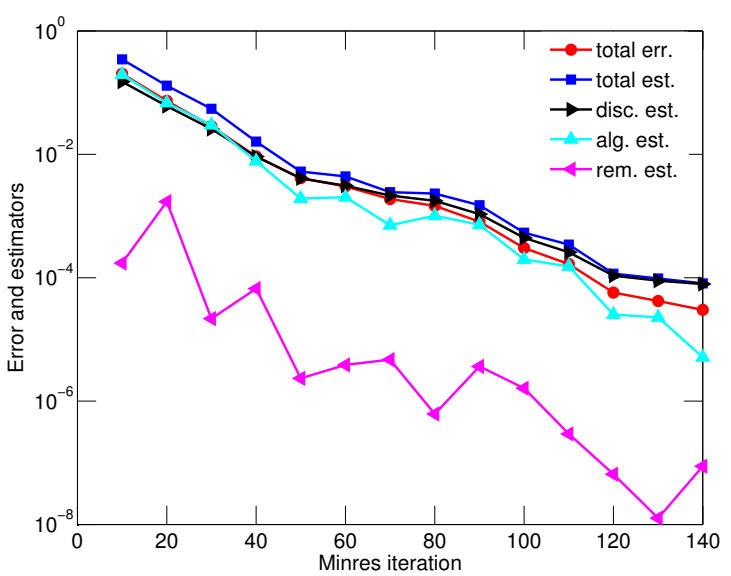

(b) Adaptive inexact MinRes, 8th mesh

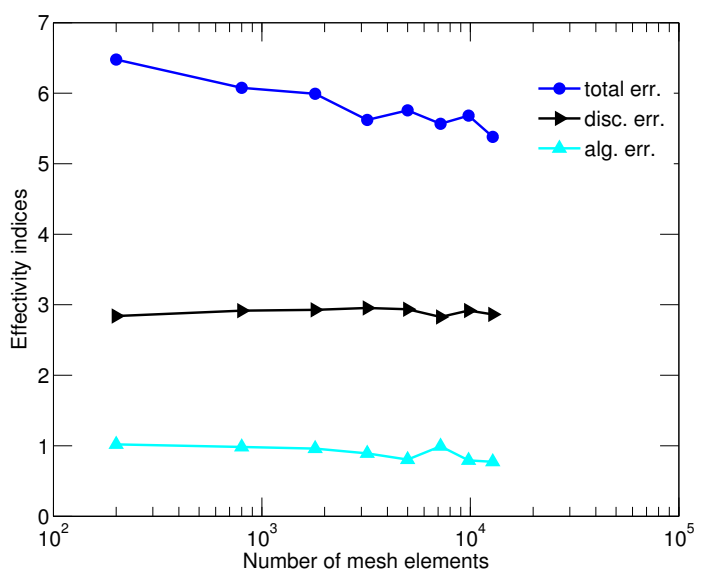

(d) Adaptive inexact MinRes, componentwise effectivity indices

Figure 7: MinRes algorithm, incomplete Cholesky factorization preconditioner 


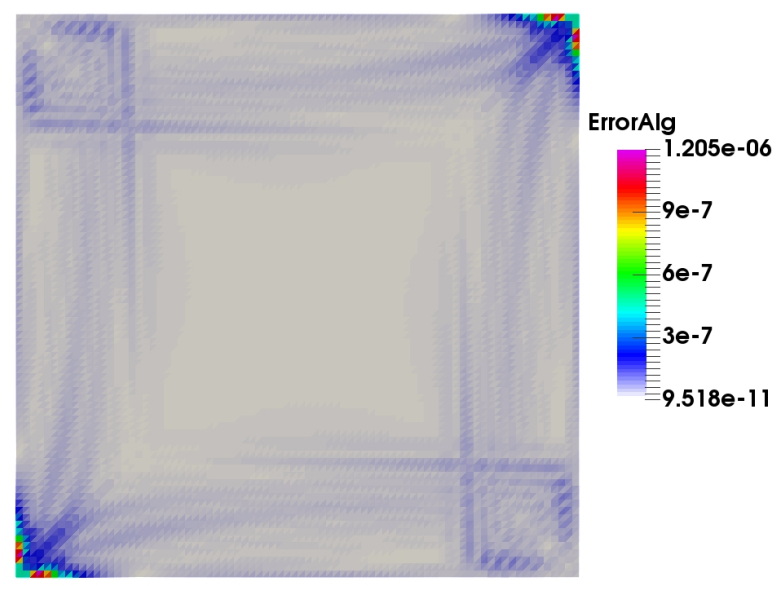

(a) Algebraic error

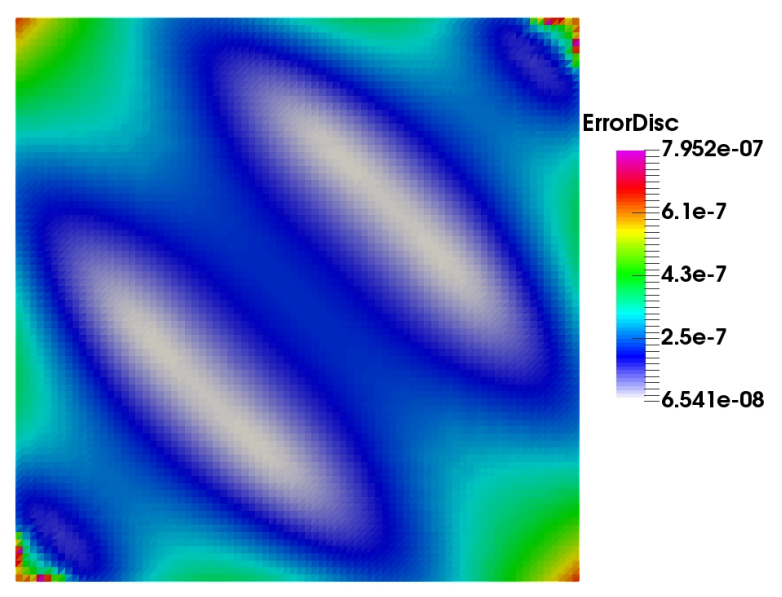

(c) Discretization error

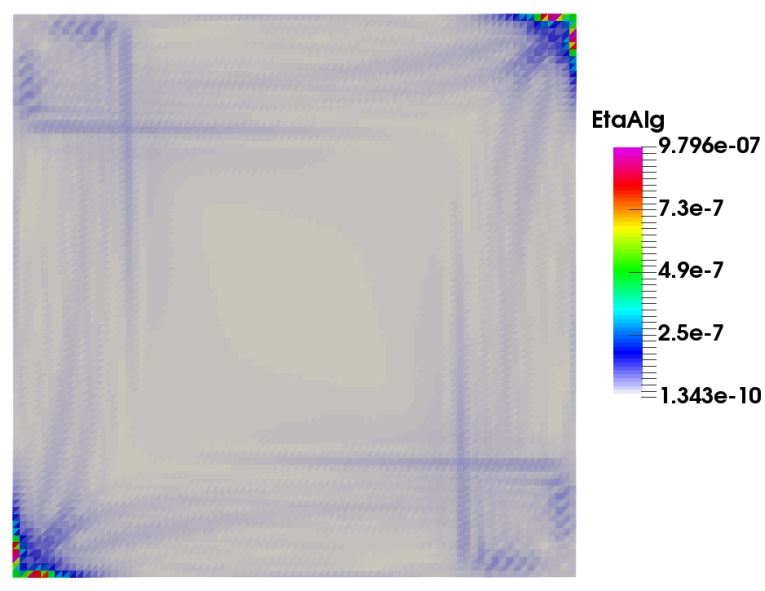

(b) Algebraic estimator

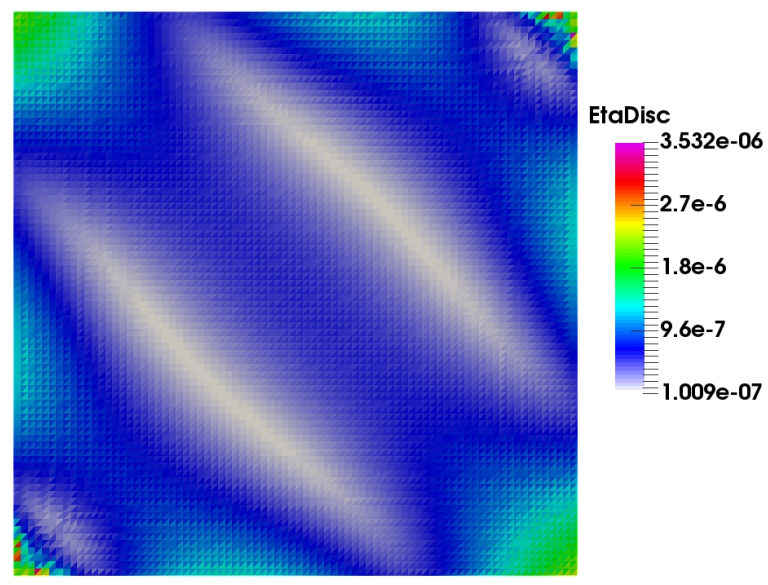

(d) Discretization estimator

Figure 8: Adaptive inexact MinRes algorithm, incomplete Cholesky factorization preconditioner. Spatial distributions of the different error components and corresponding estimates, 8th mesh 


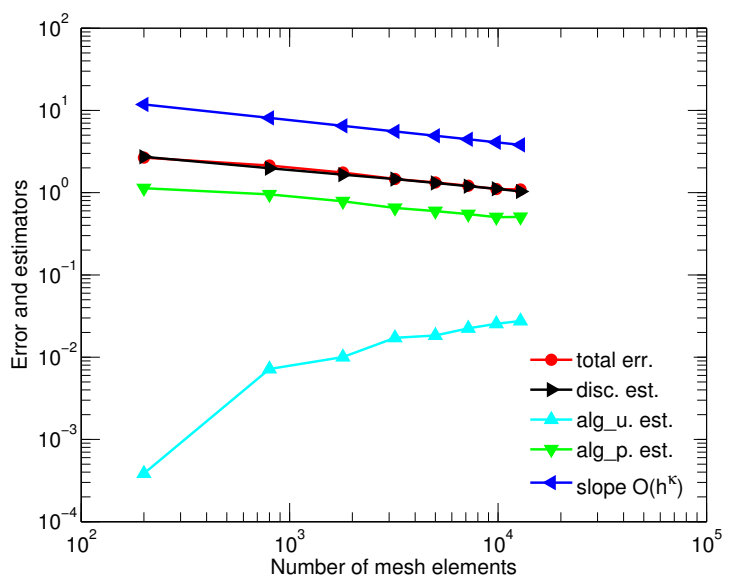

(a) Error and estimators, uniform refinement

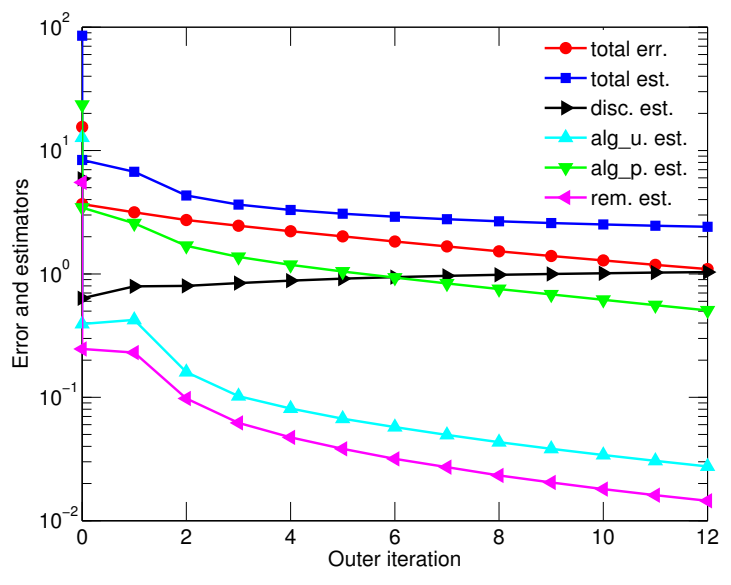

(b) Error and estimators, 8th mesh

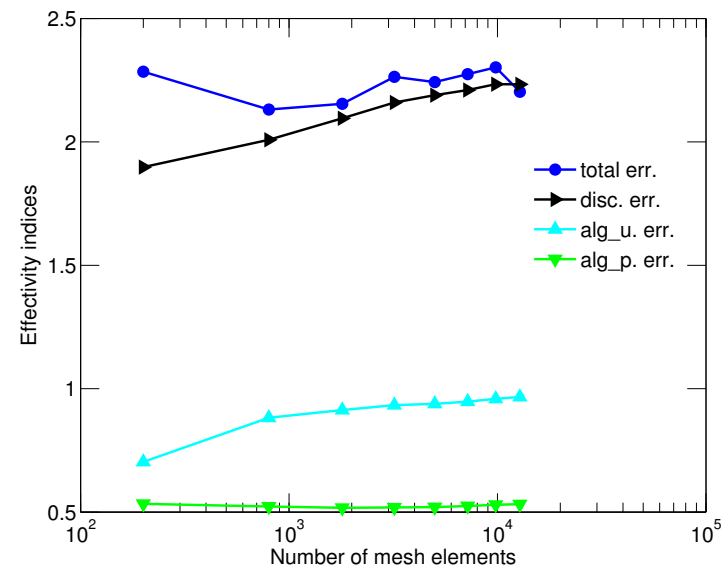

(c) Componentwise effectivity indices

Figure 9: Adaptive inexact Uzawa algorithm, CG with incomplete Cholesky factorization preconditioner 


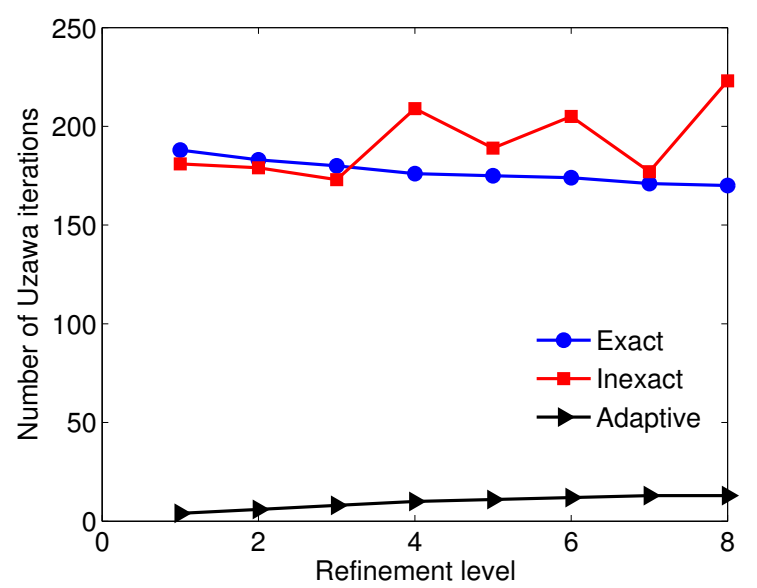

(a) Uzawa iterations per refinement level

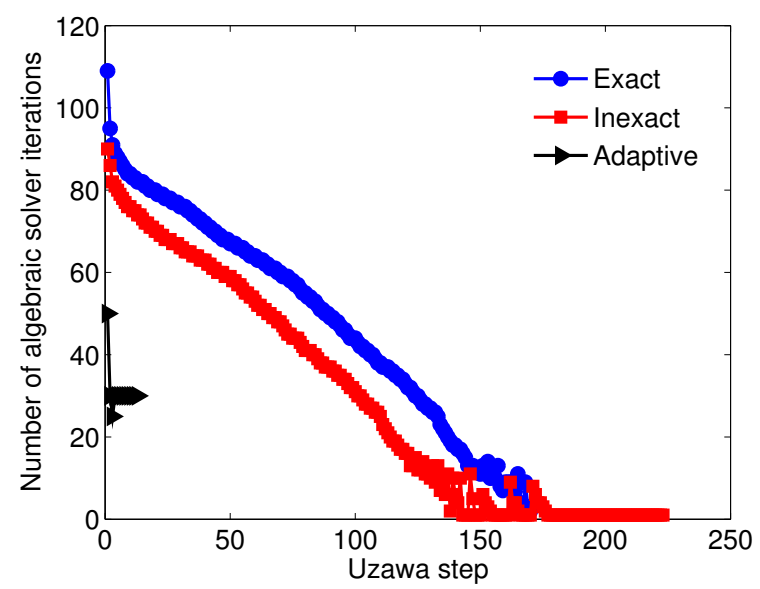

(b) CG iterations per Uzawa step, 8th mesh

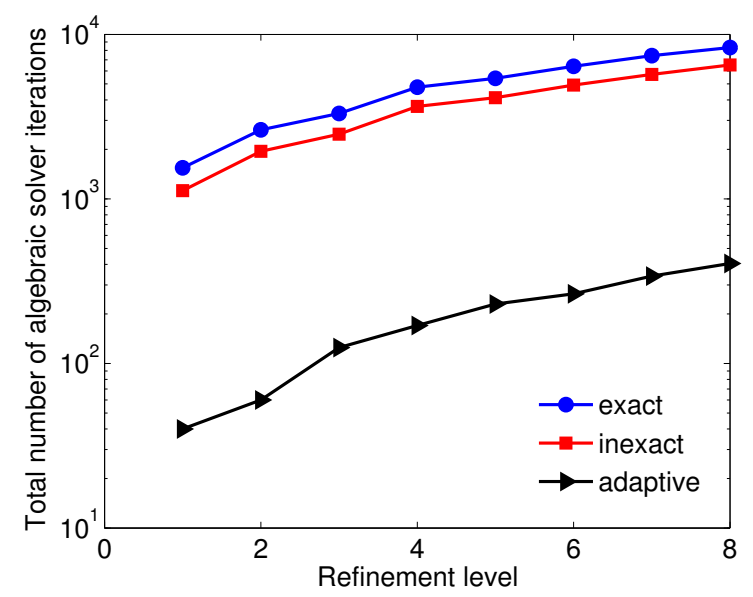

(c) CG iterations per refinement level

Figure 10: Number of preconditioned CG and Uzawa iterations 


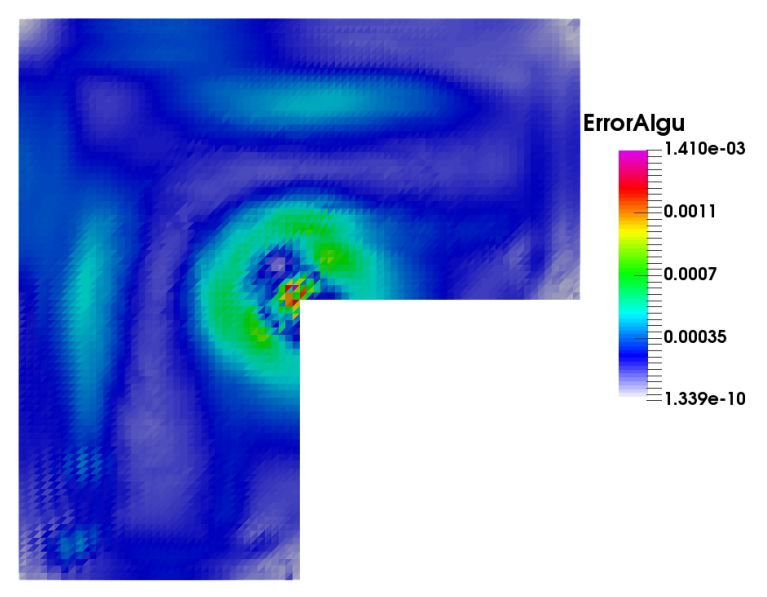

(a) Algebraic error

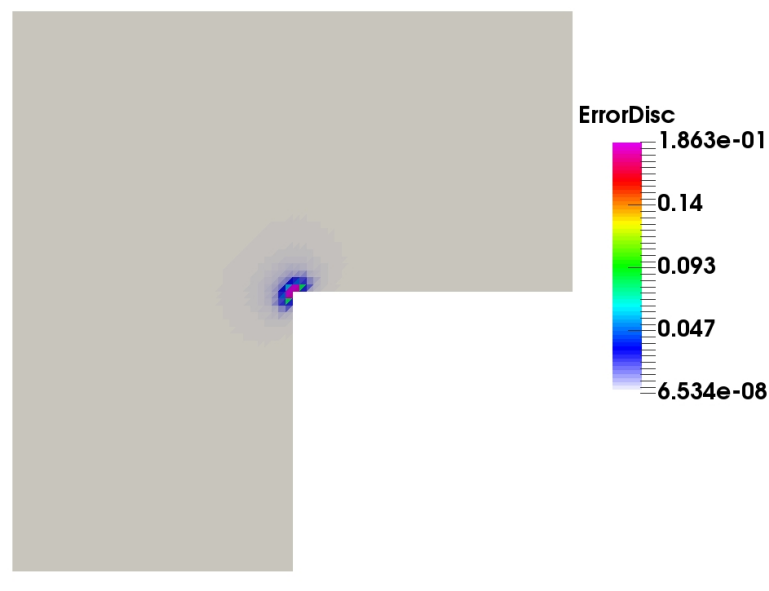

(c) Discretization error

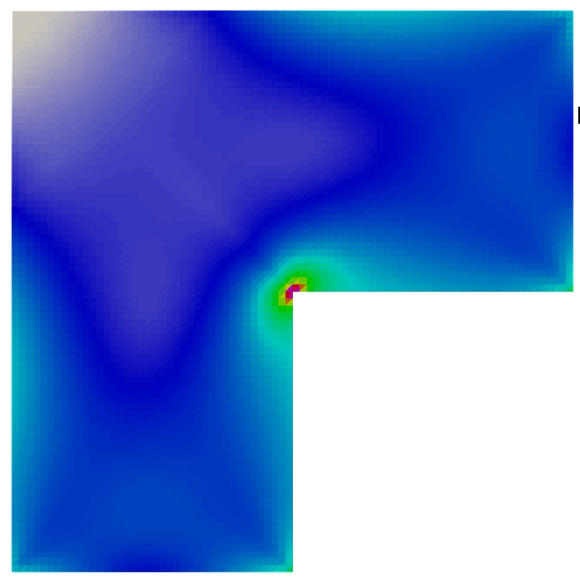

(e) Uzawa error

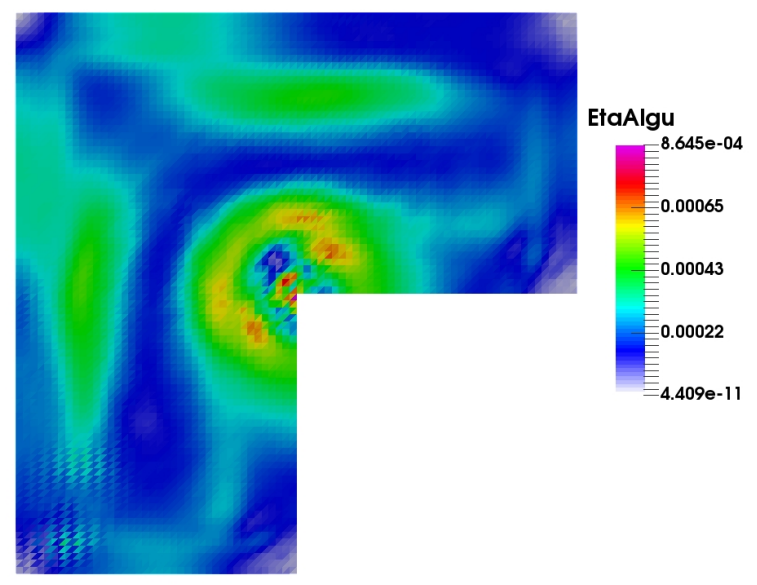

(b) Algebraic estimator

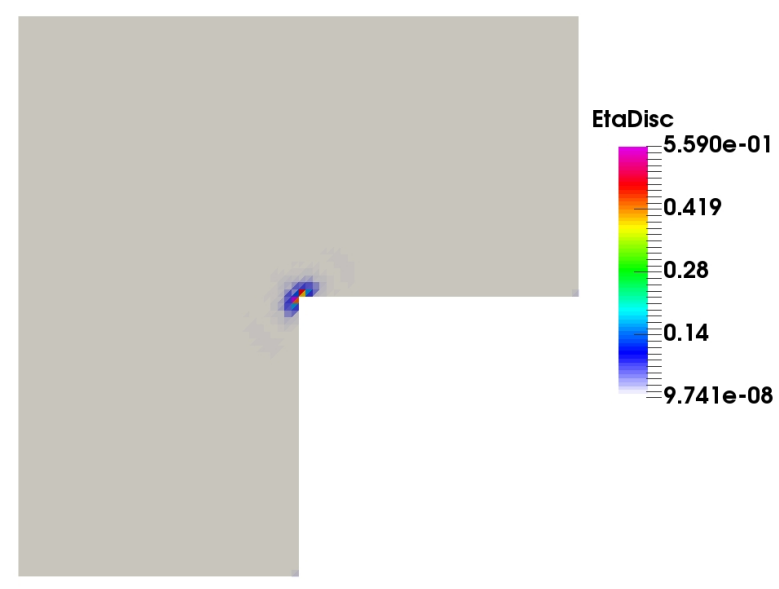

(d) Discretization estimator

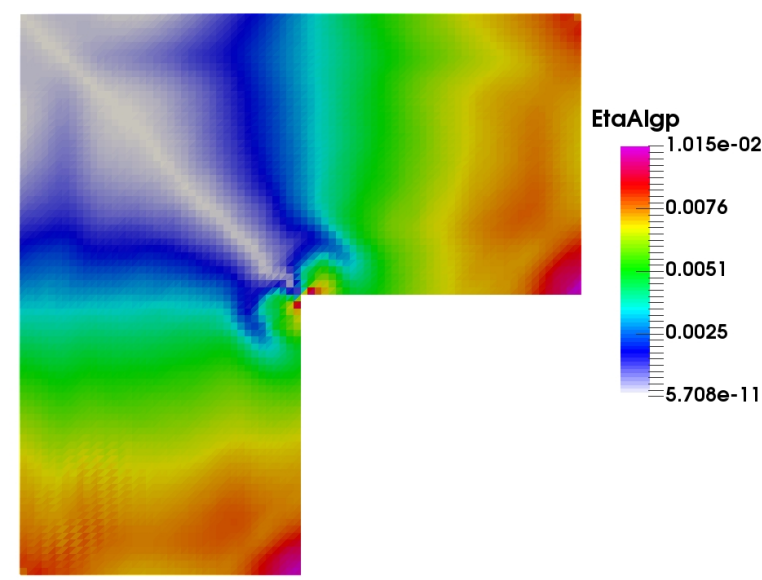

(f) Uzawa estimator

Figure 11: Adaptive inexact preconditioned CG-Uzawa algorithm. Spatial distributions of the different error components and corresponding estimates, 8 th mesh 


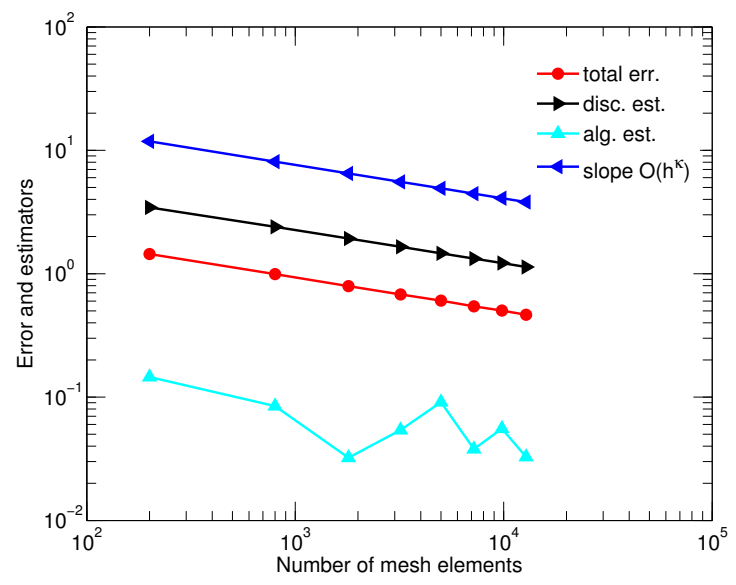

(a) Adaptive inexact MinRes, un. refinement

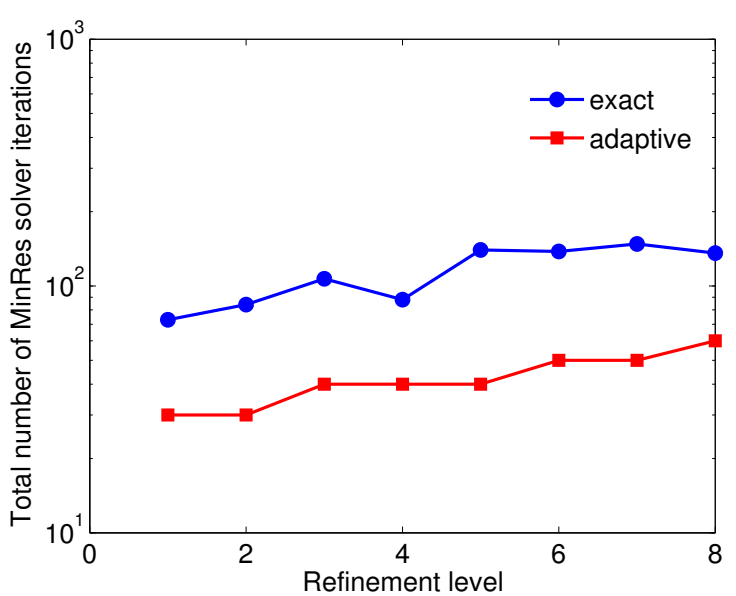

(c) MinRes iterations per refinement level

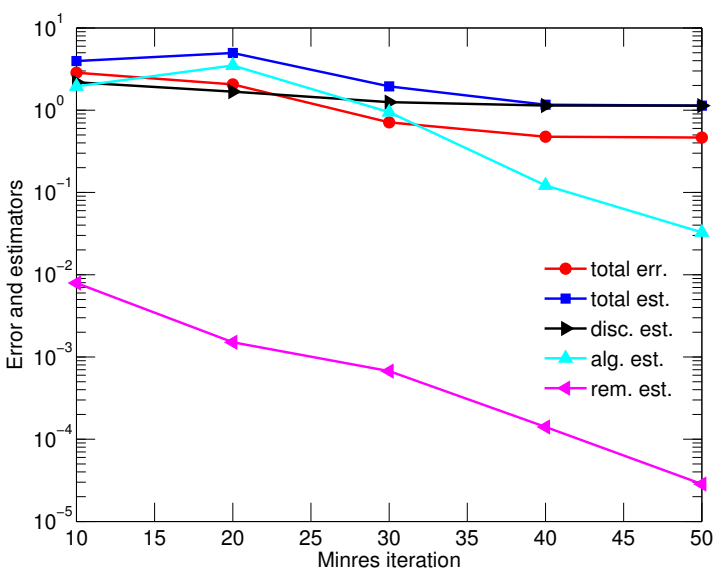

(b) Adaptive inexact MinRes, 8th mesh

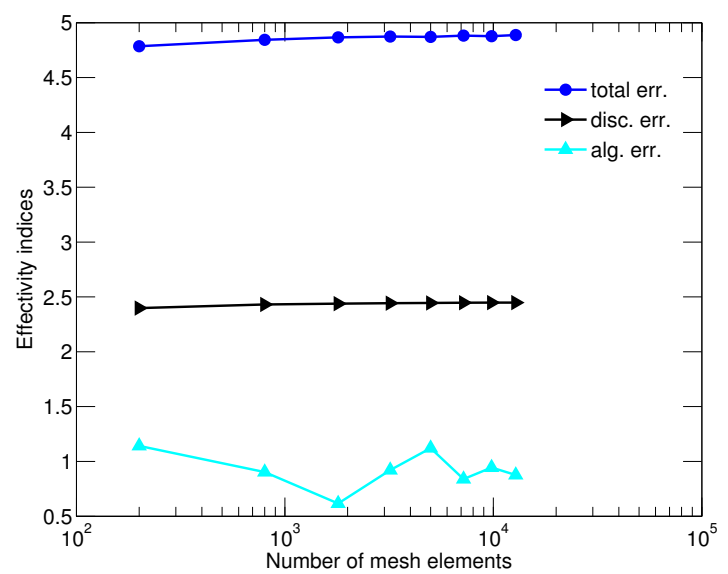

(d) Adaptive inexact MinRes, componentwise effectivity indices

Figure 12: MinRes algorithm, incomplete Cholesky factorization preconditioner 


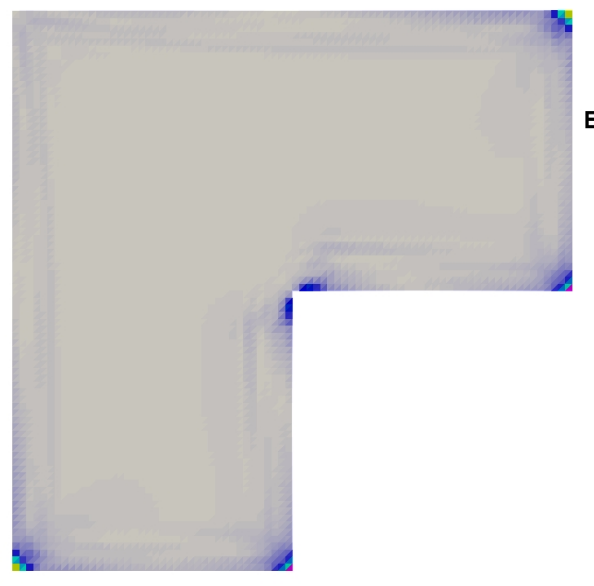

(a) Algebraic error

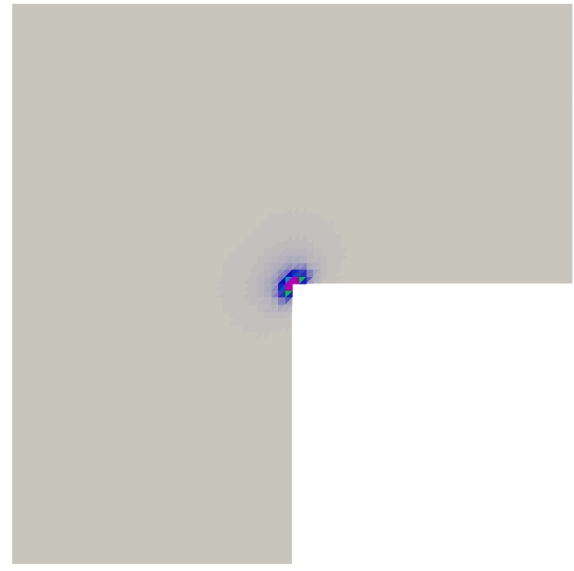

(c) Discretization error

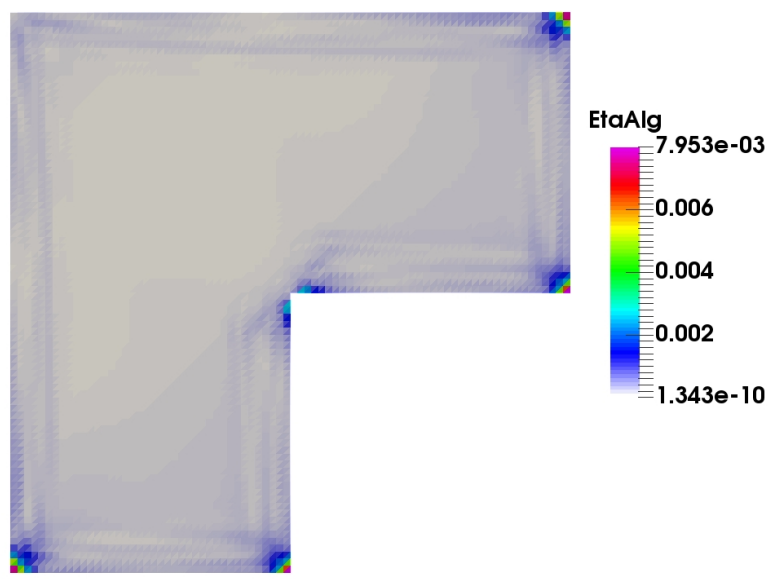

(b) Algebraic estimator

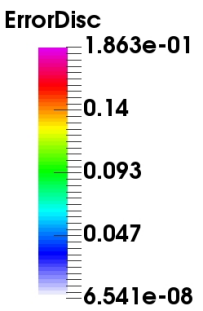

(d) Discretization estimator

Figure 13: Adaptive inexact MinRes algorithm, incomplete Cholesky factorization preconditioner. Spatial distributions of the different error components and corresponding estimates, 8th mesh 